\title{
An integrative ChIP-chip and gene expression profiling to model
} SMAD regulatory modules

\author{
Huaxia Qin $^{\dagger 1}$, Michael WY Chan ${ }^{\dagger 1,5}$, Sandya Liyanarachchi ${ }^{1}$, Curtis Balch ${ }^{4}$, \\ Dustin Potter ${ }^{1}$, Irene J Souriraj ${ }^{1}$, Alfred SL Cheng ${ }^{1,6}$, Francisco J Agosto- \\ Perez ${ }^{1}$, Elena V Nikonova7, Pearlly S Yan¹, Huey-Jen Lin², \\ Kenneth P Nephew ${ }^{4}$, Joel H Saltz ${ }^{3}$, Louise C Showe ${ }^{7}$, Tim HM Huang ${ }^{1}$ and \\ Ramana V Davuluri*1,7
}

Address: ${ }^{1}$ Human Cancer Genetics Program, Department of Molecular Virology, Immunology, and Medical Genetics, The Ohio State University, Columbus, OH 43210, USA, ²Division of Medical Technology, School of Allied Medical Professions, The Ohio State University, Columbus, OH 43210, USA, ${ }^{3}$ Department of Biomedical Informatics, The Ohio State University, Columbus, OH 43210, USA, ${ }^{4}$ Medical Sciences, Indiana University School of Medicine, Bloomington, IN 47405, USA, ${ }^{5}$ Department of Life Science and Institute of Molecular Biology, National Chung Cheng University, Min-Hsiung, Chia-Yi 621, Taiwan, Republic of China, ${ }^{\text {In }}$, Hong Kong SAR, PR China and ${ }^{7}$ Center for Systems and Computational Biology, Molecular and Cellular Oncogenesis Program, The Wistar Institute, Philadelphia, PA, USA

Email: Huaxia Qin - huaxia.qin@gmail.com; Michael WY Chan - chanmwy@gmail.com;

Sandya Liyanarachchi - sandya.liyanarachchi@osumc.edu; Curtis Balch - rbalch@indiana.edu; Dustin Potter - dustin.potter@osumc.edu; Irene J Souriraj - irene.pathy@gmail.com; Alfred SL Cheng - alfredcheng@cuhk.edu.hk; Francisco J Agosto-Perez - gagosto@wistar.org; Elena V Nikonova - enikonova@wistar.org; Pearlly S Yan - pearlly.yan@osumc.edu; Huey-Jen Lin - lin.661@osu.edu;

Kenneth P Nephew - knephew@indiana.edu; Joel H Saltz - joel.saltz@osumc.edu; Louise C Showe - lshowe@wistar.org; Tim HM Huang - tim.huang@osumc.edu; Ramana V Davuluri* - rdavuluri@wistar.org

* Corresponding author †Equal contributors

Published: 17 July 2009

BMC Systems Biology 2009, 3:73 doi:10.1186/1752-0509-3-73

This article is available from: http://www.biomedcentral.com/1752-0509/3/73

(c) 2009 Qin et al; licensee BioMed Central Ltd.

This is an Open Access article distributed under the terms of the Creative Commons Attribution License (http://creativecommons.org/licenses/by/2.0), which permits unrestricted use, distribution, and reproduction in any medium, provided the original work is properly cited.
Received: 26 August 2008

Accepted: 17 July 2009

\begin{abstract}
Background: The TGF- $\beta$ /SMAD pathway is part of a broader signaling network in which crosstalk between pathways occurs. While the molecular mechanisms of TGF- $\beta / S M A D$ signaling pathway have been studied in detail, the global networks downstream of SMAD remain largely unknown. The regulatory effect of SMAD complex likely depends on transcriptional modules, in which the SMAD binding elements and partner transcription factor binding sites (SMAD modules) are present in specific context.

Results: To address this question and develop a computational model for SMAD modules, we simultaneously performed chromatin immunoprecipitation followed by microarray analysis (ChIPchip) and mRNA expression profiling to identify TGF- $\beta / S M A D$ regulated and synchronously coexpressed gene sets in ovarian surface epithelium. Intersecting the ChIP-chip and gene expression data yielded 150 direct targets, of which 14I were grouped into 3 co-expressed gene sets (sustained up-regulated, transient up-regulated and down-regulated), based on their temporal changes in expression after TGF- $\beta$ activation. We developed a data-mining method driven by the Random Forest algorithm to model SMAD transcriptional modules in the target sequences. The predicted SMAD modules contain SMAD binding element and up to 2 of 7 other transcription factor binding sites (E2F, P53, LEFI, ELKI, COUPTF, PAX4 and DRI).
\end{abstract}


Conclusion: Together, the computational results further the understanding of the interactions between SMAD and other transcription factors at specific target promoters, and provide the basis for more targeted experimental verification of the co-regulatory modules.

\section{Background}

SMAD transcription factors are the core members of transforming growth factor $\beta$ (TGF- $\beta$ ) pathway, which has been implicated in the regulation of cell growth, differentiation, apoptosis and specification of developmental fate [1]. SMADs transmit signals from cell surface receptors to the nucleus in response to TGF- $\beta$. The general molecular mechanisms of the TGF- $\beta /$ SMAD pathway from the cell membrane to the formation of a SMAD complex in the nucleus are fairly well established. Briefly, TGF- $\beta$ elicits its molecular actions by binding to trans-membrane receptors, TGFBR1 and TGFBR2, which form an oligomeric complex and then transmit the signal into the cell via phosphorylation of SMAD2/3 proteins. Phosphorylated SMAD2/3 forms dimers or trimers with another protein, SMAD4, and this resultant SMAD complex is then translocated to the nucleus where it interacts with other DNAbinding co-regulators to modulate the transcription of TGF- $\beta /$ SMAD target genes [1-3].

The TGF- $\beta$ stimulated SMAD3/4 binds to 5'-GTCT-3', or its complement 5'-AGAC-3', called SMAD-BindingElement (SBE), with very low affinity [4]. It was initially thought that the presence of multiple SBEs in the target promoters likely enables tight binding, since activated SMAD complexes consist of SMAD oligomers. However, known SMAD target promoters seldom contain SBE concatemers, and those that contain up to four SBEs still require cooperating factors for effective DNA binding [5]. The list of DNA-binding SMAD partners, such as E2F1 [6], AP2 [7], PBX1 [8], OCT1 [9] and p300/CBP [10], is rapidly growing, and it is now believed that the high-affinity binding of the SMAD complex occurs through the incorporation of one or more different DNA-binding cofactors into the complex. Hence, the net effect of SMAD complex likely depends on transcriptional modules, in which the SBEs and partner transcription factor binding sites (TFBSs) are present in specific context. This mode of interaction provides a basis for high affinity and selectivity of target gene recognition and allows for the differential action of TGF- $\beta$ in different cell types [11]. Thus understanding the complex nature of TGF- $\beta /$ SMAD signaling requires knowing not only the set of genes bound and regulated by SMAD, but also its interacting transcription factors (together referred as SMAD modules) and the promoter regions where these interactions occur.

Abnormal activation or repression of TGF- $\beta$ regulated processes is implicated in many diseases including renal, hepatic, and neurodegenerative disorders. Epithelial cells have a high turnover and their progenitor cells divide continuously, making them prime targets for genetic and epigenetic changes that lead to cell transformation and tumorigenesis [12]. In cancer development and progression, the TGF- $\beta$ /SMAD signaling pathway functions as a double-edged sword, acting as a tumor suppressor in early tumorigenesis and as a tumor enhancer in late tumorigenesis [13]. While regulation of normal epithelial cell growth and differentiation is contingent upon appropriate up- or down-regulation of TGF- $\beta / S M A D$ responsive genes, this homeostasis is disrupted during neoplastic processes, resulting in outgrowth and invasion of transformed cells. It has been reported that neoplastic cells become non-responsive to TGF- $\beta / \mathrm{SMAD}$ signaling activation, despite the fact that upstream regulators, such as TGFBR2, remain genetically intact [14-17]. It is suggested that other aberrant events, perhaps affecting co-regulators of this growth inhibitory pathway, trigger signaling perturbations in TGF- $\beta$ /SMAD downstream targets. Although a few loci have been described in the literature, comprehensive identification of these co-regulator factors has yet to be performed [4,18-20].

In this study, we systematically identified TGF- $\beta / S M A D$ regulated and synchronously coexpressed gene sets (defined as "synexpression" groups in [1]) on genomescale by simultaneously conducting ChIP-chip (genomewide location analysis of the chromatin) and mRNA expression profiling in an immortalized ovarian surface epithelial (IOSE) cell line. The identified target genes were classified into synexpression groups based on their temporal changes in expression after the TGF- $\beta /$ SMAD signaling activation. Sequence analyses of target regions in each synexpression group revealed conserved SBEs and partner TFBSs. We applied the Random Forest (RF) [21] algorithm followed by Classification And Regression Tree (CART) [22] analysis to classify different synexpression target groups based on the presence of SBEs and binding sites of probable co-regulatory transcription factors. Several transcription factor modules were derived from this combined classification analysis, providing for the first time a com-

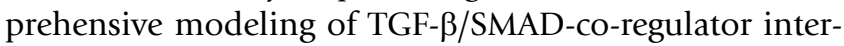
actions in ovarian surface epithelial cells and important hypotheses for further experimental work.

\section{Results \\ Identification of TGF- $\beta$ ISMAD direct target promoters by ChIP-chip}

Genome-wide discovery of TGF- $\beta /$ SMAD targets was conducted by ChIP-chip using a promoter microarray of 
$\sim 17,000$ annotated promoter regions in the human genome. To probe the arrays, we performed different ChIP assays by using an antibody against SMAD4 in immortalized ovarian surface epithelial (IOSE) cells treated with TGF- $\beta 1$ for 0 and 3 hrs. The immunoprecipitated DNA was hybridized to an Agilent $44 \mathrm{~K}$ promoter array (see Methods), and the experiments were repeated once [23]. We observed high positive correlation between the normalized log ratios (immonuprecipated DNA over total input DNA) of the biological replicate experiments, which demonstrates the reproducibility of the experiments (See Additional File 1 - Figure S1). A probe with weighted binding ratio at the 3 -hr time point above 2 ( $p$-value < 0.01 ) and having at least a $30 \%$ increase in relative binding compared to the $0 \mathrm{hr}$ time point was considered as bound (see Methods).

\section{Identification of TGF- $\beta$ ISMAD responsive genes by geneexpression profiling}

In order to identify TGF- $\beta$ transcriptionally responsive genes, we have used an Affymetrix U133 Plus 2 microarray to globally assess the gene expression in IOSE cells treated with TGF- $\beta 1$ at $0,3,6$, and 12 hrs. The experiments at each time point were repeated once, as described in Methods, and the gene expression estimates from the replicate experiments were remarkably similar (see Additional Files 2 and 3 - Figures S2 and S3 for reproducibility and clustering analyses of expression microarray). We performed Analysis of Variance to study the effect of time on gene expression and identify those genes that are differentially expressed in at least one time point (treated samples) as compared to the $0 \mathrm{hr}$ control (untreated sample).

\section{Identification of synexpression groups of TGF- $\beta$ ISMAD direct targets by intersection of ChIP-chip and gene expression data}

This analysis of ChIP-chip experiments identified 2,096 SMAD4-bound genes in IOSE cells, while expression microarrays identified 1,519 genes having expression changes following TGF- $\beta$ treatment. By combining the results from the two microarray platforms, we identified 150 differentially expressed genes that were bound by SMAD4 in their promoter regions upon activation by TGF- $\beta$ (See Additional File 4 - Table S1). One of the main goals of our study was to identify transcriptional modules containing SBE within the SMAD target promoters. Previous studies have demonstrated the utility of TFBS analyses of co-expressed gene sets to reveal cis-regulatory mechanisms in the target promoters $[24,25]$. We, therefore, performed hierarchical clustering of the expression data of the 150 genes (Figure 1) to determine different synexpression groups of TGF- $\beta /$ SMAD direct targets. To identify informative gene clusters that correspond to major synexpression groups, we pruned the hierarchical tree and identified 5 branches, including two major (Groups 2 and 4) and three minor (Groups 1, 3 and 5) groups (See Additional Files 4 and 5 - Tables S1 and S2). We considered a group as major if it contained at least 10 genes. The major groups 2 and 4 respectively correspond to up- and downregulatory expression patterns. Group 2 consists of 80 genes that showed elevated gene expression in at least one time point and group 4 consisted of 62 genes that showed decreased expression in comparison to $0 \mathrm{hr}$ time point. Group 1, 3 and 5 had one, four and three genes, respectively. We, then, focused on the two largest groups, Group 2 and 4 for further analyses to derive the SMAD modules.

Target genes within the up-regulated group were further divided into two major and one minor branch. The expression level of the 54 genes in the major branch increased after TGF- $\beta$ treatment and remained steadily high; we labeled this major branch the "sustained up-regulated group". On the other hand, the expression of the 25 genes in the middle branch had significantly increased at 3 hrs ( $\mathrm{p}$-values $<0.05$ and fold increase $>1.5$ in the comparison of $0 \mathrm{hr}$ vs $3 \mathrm{hr}$ ), and returned to baseline expression over the 12-hr period. The genes in this synexpression group were labeled the "transient up-regulated group". The bottom branch had only one gene, ATXN1, with an elevated expression at the 3 and $12 \mathrm{hr}$ time points and repressed expression at the $6 \mathrm{hr}$ time point. Overall, we have identified 3 major synexpression groups - sustained up-regulated (54 genes), transient up-regulated (25 genes) and down-regulated (62 genes).

\section{Experimental validation of TGF- $\beta$ ISMAD binding}

SMAD4 binding of 10 randomly selected loci of the 150 targets that were shown to both bind SMAD4 and change gene expression in response to TGF- $\beta$, were confirmed in individual ChIP assays. On average, greater than 1.5 foldenrichment was observed in IOSE cells after 3 hrs treatment with TGF- $\beta 1$ (Figure 2 ). RT-PCR analysis was used to confirm altered expression of five group 2, two group 3 , and three group 4 genes at 0, 3, 6 and 12 hrs after TGF- $\beta$ stimulation (Figure 3). Specifically, we observed that the increase in expression of ADAM19, FBXO32, RunX1T1, and $D D A H$ (group 2, sustained up-regulated) was maintained at the time-course between 3 and 12 hrs after treatment, while ZNF638 (group 2, transient up-regulated) showed increased expression at the $3 \mathrm{hr}$ time point and gradual decrease to base-line level at the $12 \mathrm{hr}$ time point. Decreased expression of FRAT and CXXC6 (group 3) was observed at 3 hrs after treatment. Expression levels of these two genes tended to increase afterwards, but remained below baseline levels by 12 hrs of treatment. On the other hand, expression of NTN4, ADPN, and RGS17 (group 4) continued to decrease at 6 hrs or 12 hrs after treatment. To summarize, the overall trends of temporal changes of expression and binding by SMAD4 observed in 


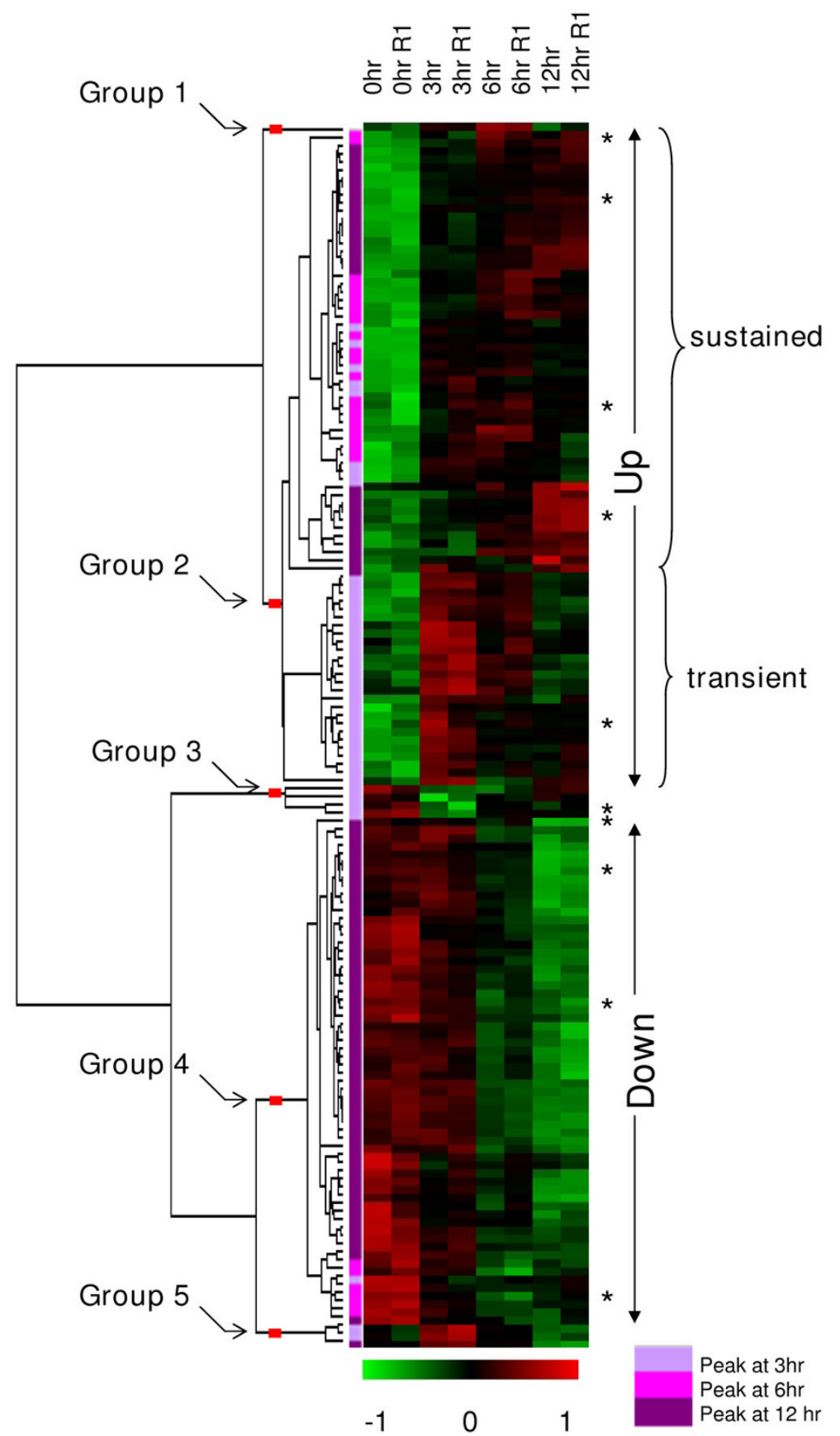

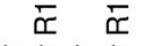

농홍호를

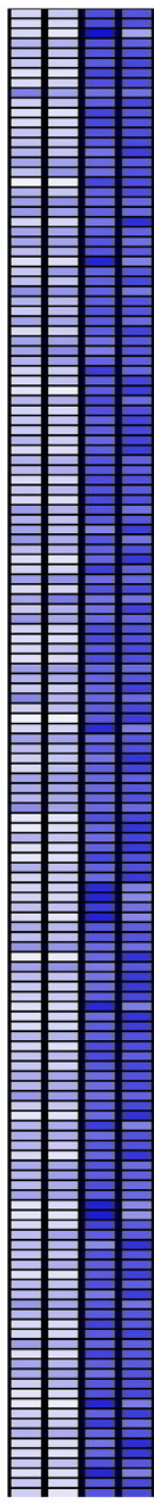

0

\section{Figure I}

Hierarchical cluster analysis of gene expression estimates from the expresssion microarrays and the heat map of binding ratios of the ChIP-chip experiments of the I50 TGF-B/SMAD target genes in IOSE cells. Genes that showed both altered expression at 3,6, or 12 hrs relative to $0 \mathrm{hr}$ (low-green and high-red) and altered binding at $3 \mathrm{hrs}$ compared to $0 \mathrm{hr}$ (low-white and high-blue) after TGF- $\beta$ signaling stimulation were shown. The expression data were median-centered and normalized to have a unit sum of squares for each gene before transforming to the color scale. Genes that were confirmed by ChIP-PCR and RT-PCR are indicated by asterisks. Results from the ANOVA analysis indicating the peak time point relative to $0 \mathrm{hr}$ is indicated by a color bar. The experiments labeled RI were biological replicates in the expression microarrays and were technical replicates in the ChIP-chip experiments. In the Chip-chip experiments, normalized binding ratios to have a unit sum of squares for each gene were used for the heatmap. 
ADAM19

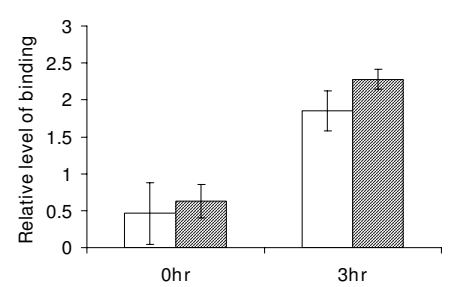

FBX032

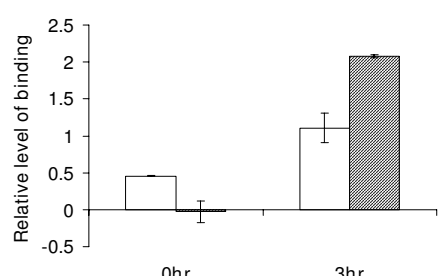

RunX1T1

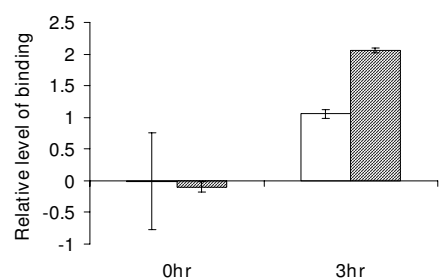

$D D A H$

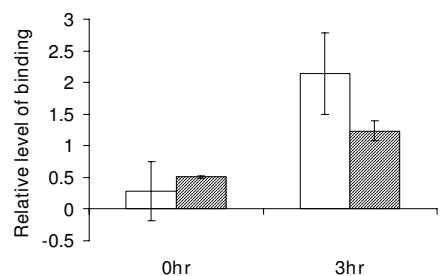

ZNF638

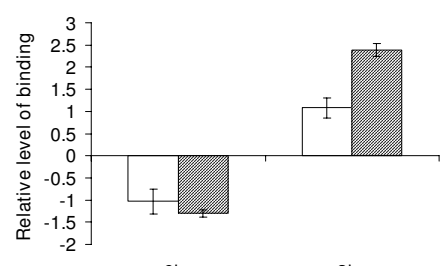

Ohr

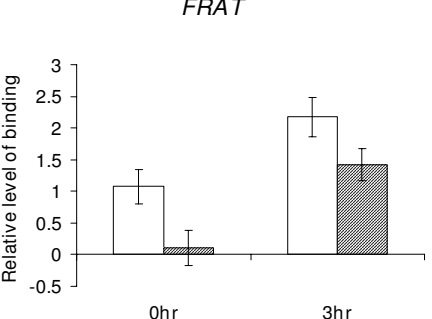

CXXC6

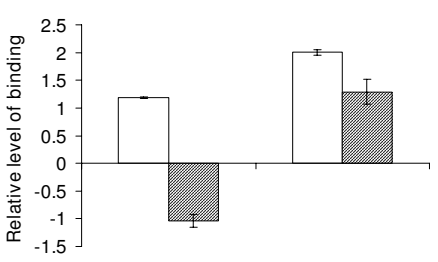

Ohr

NTN4

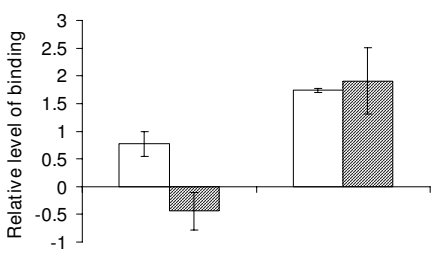

Ohr

$3 \mathrm{hr}$

ADPN

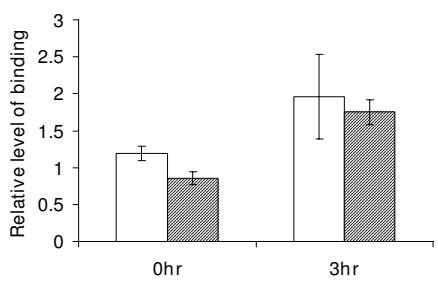

RGS17

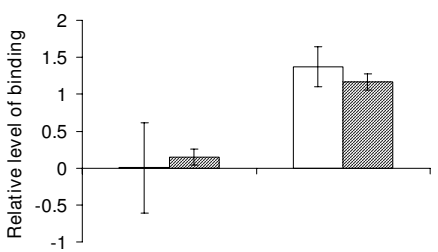

Ohr $\quad 3 h r$

\section{Figure 2}

Experimental validation of ChIP-chip results by ChIP-PCR analysis of 10 randomly selected TGF- $\beta$ /SMAD target genes. The cross-linked DNA from IOSE cells treated with TGF- $\beta$ I of 10 genes were amplified by ChIP assays and measured by a real-time PCR machine. The ChIP-PCR values were from normalized experimental results from a standard curve, which was derived from total input DNA using the same primers. The plots are in log2 scale. 
ADAM19

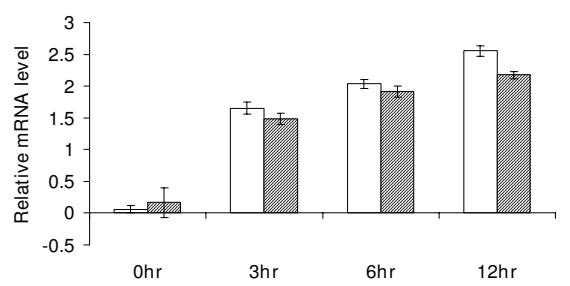

FBXO32

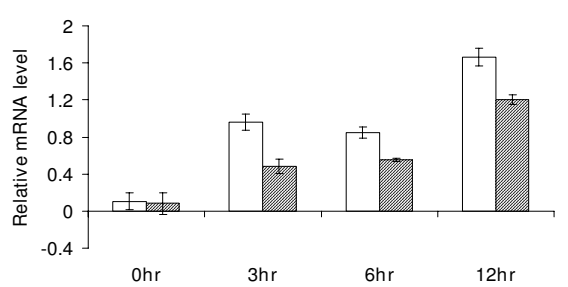

RunX1T1

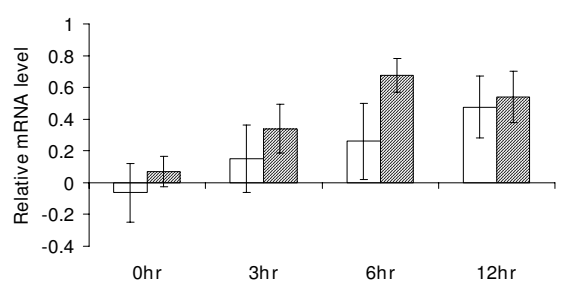

$D D A H$

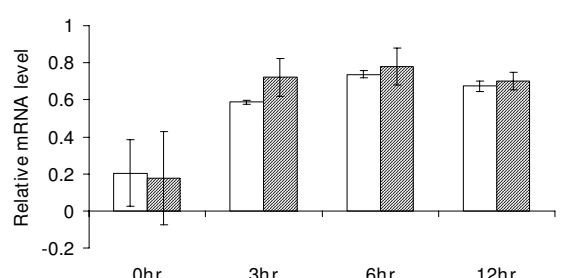

ZNF638

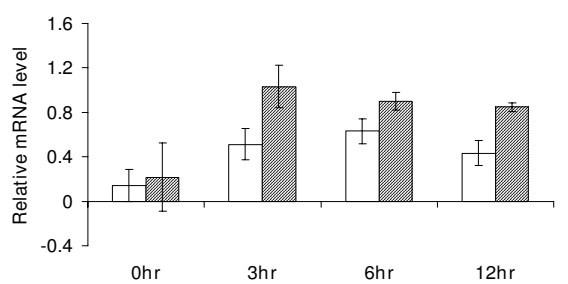

FRAT

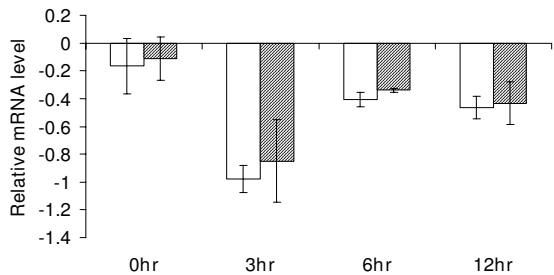

CXXC6

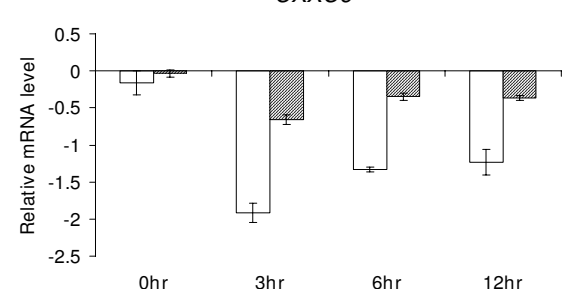

NTN4

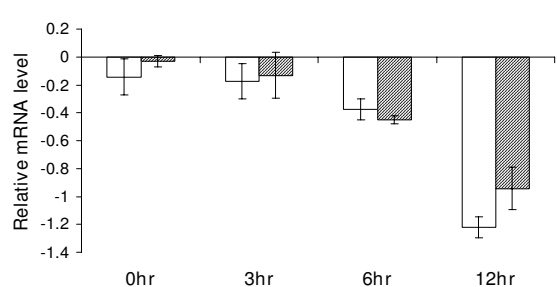

$A D P N$

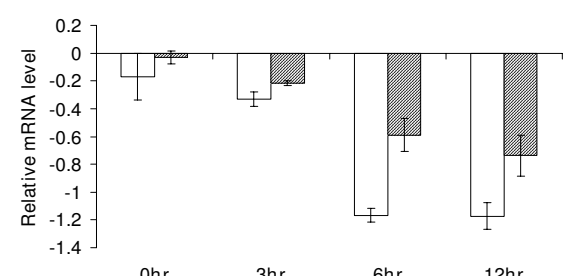

RGS17

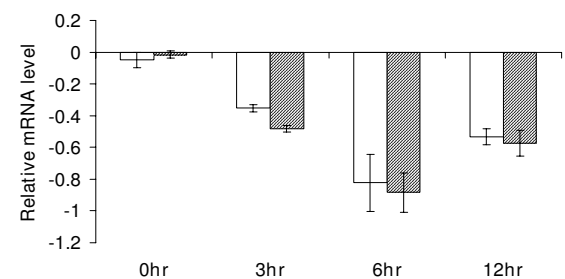

Figure 3

Experimental validation of microarray results by RT-PCR analysis of 10 randomly selected TGF- $\beta / S M A D$ target genes. The mRNAs of 10 genes from IOSE cells, treated with TGF- $\beta$ I, were amplified by RT-PCR and measured by a real-time PCR machine. The fold-change in mRNA expression for each gene was calculated by setting the expression (RT-PCR or microarray) values at $0 \mathrm{hr}$ to I. The plots are in $\log 2$ scale. 
our microarray platforms were recapitulated by the RTPCR and ChIP-PCR experiments, respectively.

\section{Random Forest variable selection followed by CART modeling identified novel co-regulatory modules in TGF- $\beta$ I SMAD-responsive genes}

In order to infer potential cis-regulatory SMAD modules and to discriminate target promoters of different synexpression gene groups, we applied classification methods driven by statistical learning approaches. For this classification analysis, we choose three major synexpression groups (sustained up-, transient up- and down-regulated from the hierarchical clustering) as different classes. The TFBSs, predicted by MATCH program, using the TRANSFAC (version 9.1) position weight matrices (PWMs), were used as the predictor variables of the classification function. Each predictor variable takes binary values 0 or 1 depending on the presence or absence of corresponding TFBS in the promoter region of interest. An attempt to classify the three synexpression groups (sustained up-, transient up- and down-regulated targets) by 3-class classifier resulted in very poor classification models (See Additional File 5 - Table S3), probably due to insufficient power as a result of the small sample sizes in each group. We, therefore, proceeded with a binary classification approach to build different classifiers for the two datasets - dataset 1 (up- vs. down-regulated targets) and dataset 2 (sustained up- vs. transient up-regulated targets).

We determined the presence of SBEs in the 150 putative targets using the position weight matrix that we developed on 67 experimentally known SBEs. The consensus sequence of SBEs is highly degenerate with a 5-bp core sequence CAGAC (Figure 4). We scanned $1 \mathrm{Kbp}$ region (500-bp on each side of the probe) around all the positive 60 -mer probes on the promoter microarray and found that $124(82.6 \%)$ of the aforementioned 150 loci contained at least one SBE (Figure 4, See Additional File 5 Table S2). The criterion set for searching SBEs was based on the upper limit of sonicated DNA fragment length (i.e., $\sim 500 \mathrm{bp}$ ) in the ChIP assay. To confirm the significant enrichment of SBEs in the target regions, we scanned for SBEs in a randomly selected 10,000 sequences of length $1,060 \mathrm{bp}$ from the human genome. We found that $71 \%$ of these regions contained at least one SBE at the same cutoff used for scanning the target regions. This suggests that there is a significant enrichment of SBEs (Fisher's exact test $p$-value 0.001 ) in the 150 putative targets in comparison to the random set of sequences of similar length.

Next, we computationally analyzed the 124 loci for the presence of other TFBSs. A 440-bp sequence region centered on one SBE was obtained. This window $(-220 \mathrm{bp}$, $+220 \mathrm{bp}$ ) was used because $220 \mathrm{bp}$ is the estimated length of DNA in one nucleosome. For sequences with more

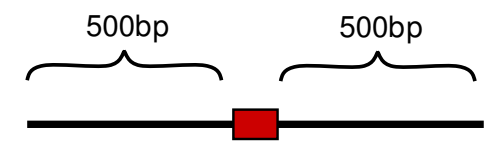

60-mer

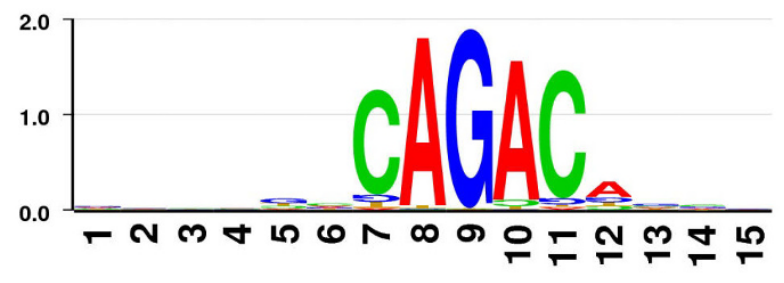

SMAD Binding Element (SBE)

\section{Figure 4}

Computational prediction of SMAD binding elements (SBEs). DNA sequences ( I,060 bp) centered around each positive 60-mer probe sequence were used to detect SBEs. The degenerate nature of the binding site is shown by the SBE sequence logo, which was based on the 67 SBEs from literature (The logo was generated on http://www.biogenio.com/logo/logo.cgi).

than one SBE, the one closest to the center of the 1,060 bp region was chosen. The MATCH program was used to predict the TFBSs [26]. These binding sites, present in at least $35 \%$ of either group (up- or down-regulated targets for dataset 1 , sustained or transient targets for dataset 2), were retained in the data matrix as predictor variables. The 35\% cut-off was arbitrarily chosen to keep the number of the predictor variables within a reasonable range. To increase our confidence, we tested and found that variation of this cut-off from $20 \%$ to $40 \%$ did not influence the outcome of the analyses. The final data-matrices contained a set of 164 and 159 TFBSs as predictor variables for dataset 1 and 2 , respectively.

We initially fitted CART and RF models to our data. A direct application of these models did not provide satisfactory prediction accuracies (Table 1 ). However, a feature of RF that is especially relevant in the current analyses is the variable importance measure, which estimates the relative importance of the TFBSs in discriminating one group from another and helps to select the TFBSs that are probably involved in the SMAD modules. RF provided a rank for each of the prediction variables based on mean decrease in accuracy of classification. We present the top 30 ranked variables identified by RF analyses in Figures 5 and 6 . We also collected transcription factors that are known to interact synergistically with SMAD from published literature and the information is presented in Table 2. It is interesting to note that more than one third of the TFs in the RF generated lists (Figures 5 \&6) are previously 
Table I: Misclassification rates by CART and RF modeling

\begin{tabular}{llll}
\hline & Number of Independent variables & Error rate \\
& & Class I & Class 2 \\
\hline Dataset I: Down/Up & & Down & Up \\
Sample Size & & 51 & 65 \\
CART & 164 & 0.41 & 0.46 \\
RF & 164 & 0.59 & 0.31 \\
RF + CART & 4 & $\mathbf{0 . 3 7}$ & $\mathbf{0 . 2 3}$ \\
Dataset 2: Transient/Sustained & & Transient & Sustained \\
Sample Size & 159 & 23 & 41 \\
CART & 159 & 0.22 & 0.68 \\
RF & 3 & 0.86 & 0.19 \\
RF + CART & & $\mathbf{0 . 1 7}$ & $\mathbf{0 . 2 7}$ \\
\hline
\end{tabular}

For each dataset, the synexpression group labeling was the dependent variable and the TFBSs were the independent variables. CART model was derived by using Gini splitting criterion, equal prior setting, unitary cost and a 10 -fold cross validation. The best tree was selected by minimum cost. The error rates were the rates on the test sample by cross validation. RF was run with stratified sampling with an equal sample size for both classes, whereas the sample size was set to the one of the class with smaller number of observations. The error rates were the average of out-of-bag error rates of 100 runs of RF, each with 1000 trees. RF + CART was to build a CART model on the top most important variables selected by RF. For both datasets, RF + CART provided the best classification results with lowest misclassification rates.

known to interact cooperatively with SMAD. On the other hand, of the 22 known SMAD co-regulators in Table 2, $27 \%$ and $45 \%$ were respectively represented in the top 30 TFBSs selected by RF from datasets 1 and 2 (See Additional File 5 - Table S4). We then fitted the CART model on subsets of these pre-selected variables. Using this implementation, the misclassification rates were dramatically improved in all the cases (Table 1). Although some misclassification rates were still high, the classification model and the RF generated lists provided an important first step in the direction of predicting cis-regulatory modules involving SMAD.

The binary trees constructed by CART are presented in Figures 7 and 8 . Six TGF- $\beta /$ SMAD co-regulators that could influence the expression status of target genes in each synexpression group, LEF1, ELK1, COUPTF, E2F, P53 and PAX4, were identified. The presence or absence of four of these TFBSs (LEF1, ELK1, COUPTF and E2F) distinguished between up- and down-regulated TGF- $\beta / S M A D$ targets, and binding of P53 and PAX4 was associated with up-regulated genes (sustained and transient groups). The DR1 in the CART tree that discriminates the subgroups of up-regulated targets stands for Direct Repeat 1, a DNA site bound by transcription factors PPAR, HNF-4, COUPTF and RAR from the family of thyroid hormone receptorlike factors. Table S5 (See Additional File 6) contains the list of modules and their target genes. Interestingly, several up-regulated target genes (PTHLH, DKK1 and CFLAR), predicted to have a P53 binding site, have been validated experimentally by others, indicating that our prediction approach is correct for these three genes [2729]. To further validate our model, we performed RNAi knock-down experiments for ADAM19 [30], a gene predicted to have SMAD4 and E2F binding sites and whose expression is upregulated in normal ovarian epithelial cells (Figure 3). However, only a slight decrease in ADAM19 expression was observed after knock-down of SMAD4 in TGF- $\beta$-treated IOSE, suggesting that additional factors regulate expression of this SMAD4 target gene [30]. In this regard, our future experiments will investigate a role for E2F play in the control of ADAM19 expression.

\section{Functional analyses of TGF- $\beta$ ISMAD-responsive genes}

We performed Ingenuity Pathway Analysis (Ingenuity ${ }^{\circledR}$ Systems, http://www.ingenuity.com, IPA 6.0) in order to find significant molecular functional categories in SAMDtarget gene set and transform the target genes into a set of relevant networks by using literature-based records that are maintained in the Ingenuity Pathway Knowledge Base. We first performed IPA analyses independently on SMADresponsive genes (from Affymetrix microarray analysis) and SMAD target genes (from ChIP-chip data analysis). The analyses produced 20 significant molecular and cellular functions (See Additional File 7 - Figure S4) that are significantly enriched in both gene sets. We found considerable overlap in the predicted pathways from both the data sets, and Wnt/ $\beta$-catenin signaling pathway as one of the most significant pathways (Figure 9 and Table 3 ). Further, LEF1, an important transcription factor of $\beta$-catenin pathway, was predicted as a co-regulator of SMAD in our predicted SMAD modules. The cooperation between TGF$\beta$ and Wnt signaling pathways was also shown by several of earlier studies [31].

\section{Discussion}

Through the interactions of different co-regulators, specific transcription factors can regulate different cellular processes which sometimes lead to opposite downstream effects [32,33]. SMAD transcription factors rely on tran- 
Table 2: List of transcription factors that are known to interact synergistically with SMAD (collected from literature search)

\begin{tabular}{|c|c|}
\hline Cofactors & Target genes \\
\hline GATA4 & IAP, IFABP [74]; INHA [75] \\
\hline $\mathrm{C} / \mathrm{EBP} \beta, \mathrm{E} 2 \mathrm{~F} 4 / 5$, FoxO & pI5INK4b [76]; c-MYC, p2 ICipI, GADD45A, GADD45B, IERI, CTGF, JAGI, LEMD3, SGK, CDC42EP3, and OVOLI [77] \\
\hline STAT3 & GFAP; HP [78-80] \\
\hline LEF/TCF & MYC [8I]; Xtwn [52] \\
\hline TCF/ $\beta$-catenin, API & Gastrin $[82,83]$ \\
\hline MYOCD & SM22alpha, Tagln [84] \\
\hline P53 & AFP [85], AFP [56] \\
\hline sox9 & COL2AI[86] \\
\hline COUPTF & COL7AI[54]. \\
\hline SPI, SP3 & LPL [87]; vimentin [88] \\
\hline ETS-I & CCN2 [89] \\
\hline NFKappaB and API & IL6 [90] \\
\hline $\mathrm{HIFI} \alpha$ & VEGF [9I] \\
\hline AP-I & $E T I[92]$ \\
\hline HNF-4 & apolipoprotein C-III [58] \\
\hline TFE-3 & SERPINEI (PAI-I) [93] \\
\hline MITF & mmcp-7 [94] \\
\hline OCT-I & GATA2 $[95,9]$ \\
\hline AP-2 & Col7al[7] \\
\hline $\mathrm{RAR} \gamma$ & human gene promoter construct [59] \\
\hline
\end{tabular}

scription cofactors for appropriate activation or repression of target genes in response to TGF- $\beta$. TGF- $\beta /$ SMAD signaling pathway is important for growth inhibition in normal ovarian epithelium, thus signaling disruption may lead to ovarian tumorigenesis [13]. Although primary ovarian cancer retains TGF- $\beta$-mediated growth inhibition, studies demonstrated that most ovarian cancer are non-responsive to TGF- $\beta$ signaling pathway $[15,34]$. The cell line that we used in this study is responsive to TGF- $\beta$ thus allowing us to evaluate the disruption of TGF- $\beta$ signaling pathway in ovarian cancer. While most of the recent studies used expression microarrays to interrogate TGF- $\beta$ targets in a particular system, those approaches cannot differentiate between direct and in-direct TGF- $\beta /$ SMAD4 targets
$[35,36]$. In our study, we combined both ChIP-chip and expression microarray data to identify TGF- $\beta$ /SMAD4 targets. This approach provides important information regarding the direct TGF- $\beta /$ SMAD 4 targets as they relate to ovarian biology. The regulatory module that we identified may also be important in understanding the disruption of TGF- $\beta$ signaling in ovarian cancer. Only 17 out of the 150 TGF- $\beta / S M A D$ targets were previously known to respond to TGF- $\beta$ in various systems (See Additional File 4 - Table S1). Thus, more than $90 \%$ of the targets identified in this study are novel targets.

To gain further insight into the potential biological relevance of these newly identified TGF- $\beta /$ SMAD targets, we 


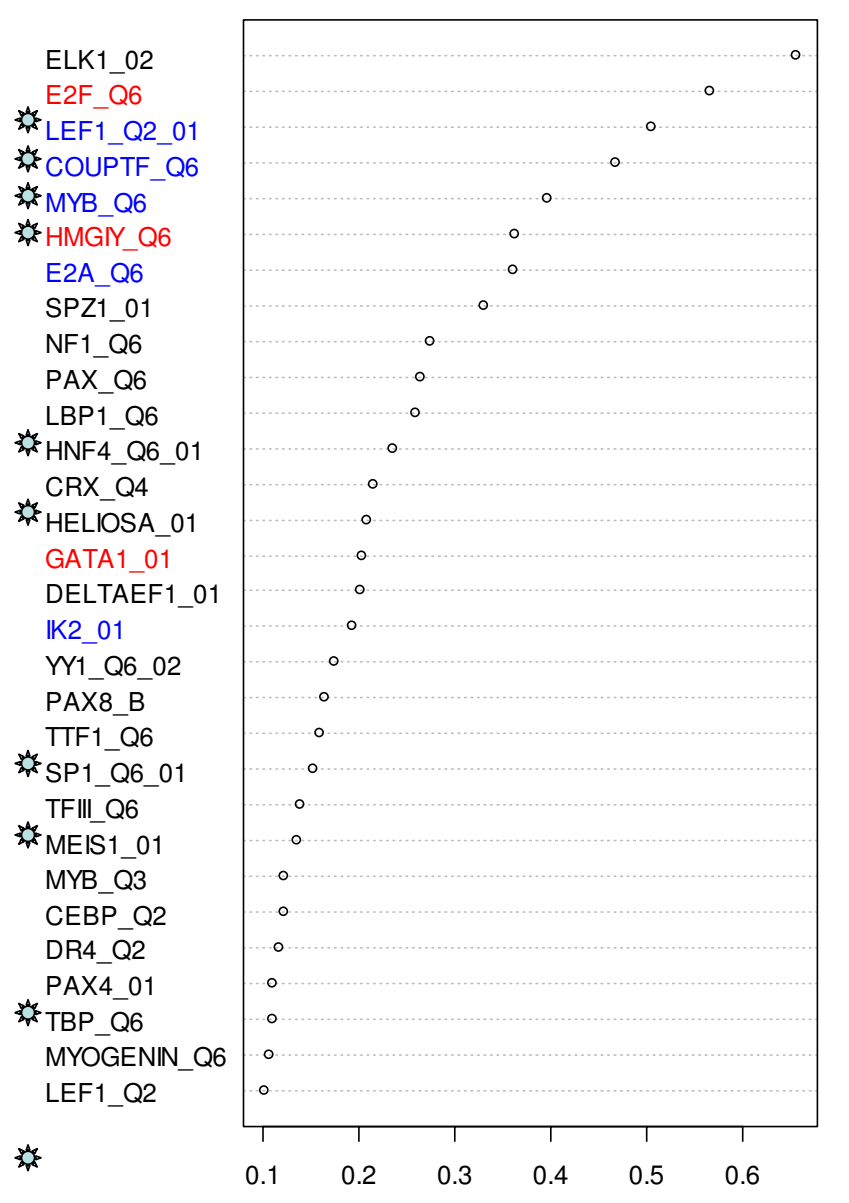

Mean Decrease in Accuracy

\section{Figure 5}

Top 30 TFBSs selected by RF and their mean decrease in accuracy in up- vs. down-regulated SAMD target genes. The mean decrease in accuracy was an average of 100 runs of RF. Experimentally known SMAD interacting TFBSs are marked with. Statistically significant $(p-$ value $<0.05$ by Fisher's exact test) over-represented TFBSs are labeled in red and blue colors: red, over-represented in up- compared to down-regulated; Blue, over-represented in down- compared with up-regulated genes.

classified the 150 targets by biological functions. This analysis revealed that the majority of the targets are related to either signaling pathways or play a role in transcriptional regulation (See Additional File 7 - Figure S4). For example, expression of the kit ligand, KITLG, is downregulated after addition of TGF- $\beta$, a result consistent with a previous study demonstrating that treatment of rat ovarian surface epithelial cells with TGF- $\beta$ results in KITLG downregulation [37]. Interestingly, KITLG is an important regulator of ovarian surface epithelial cell growth, and up-
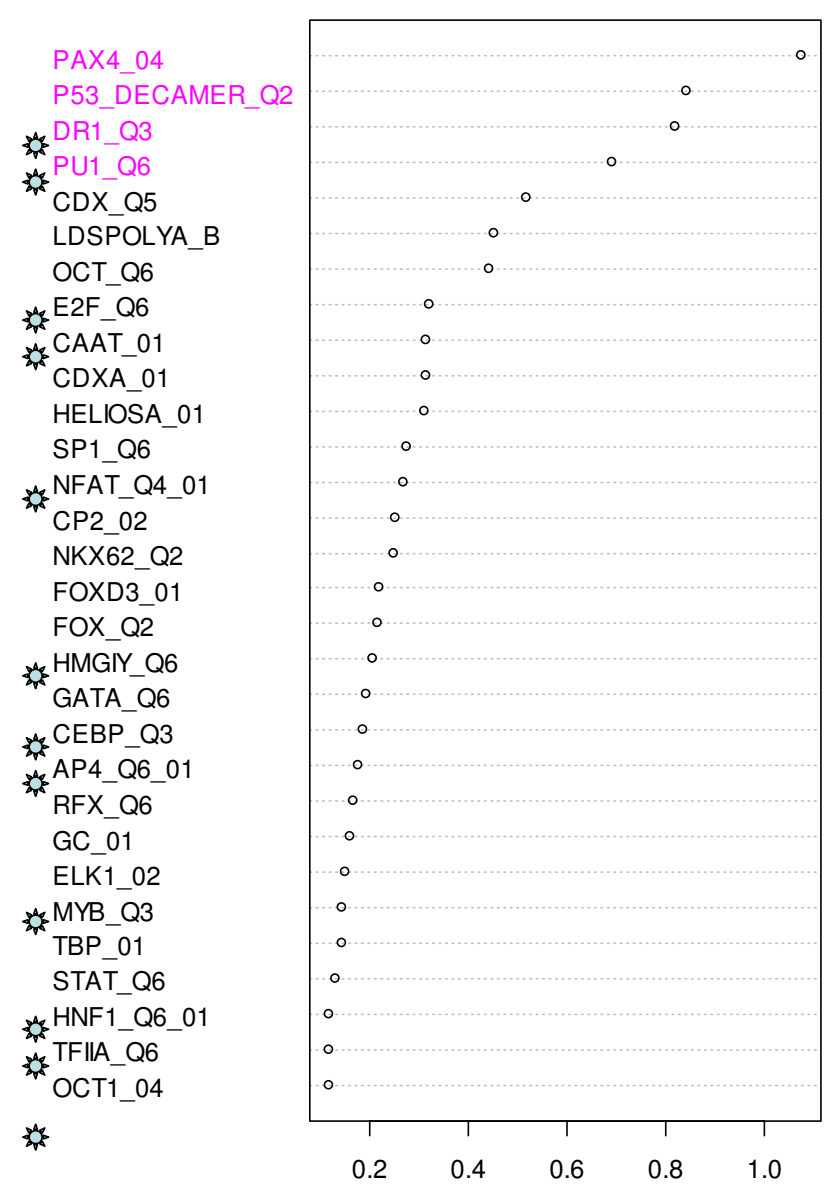

Mean Decrease in Accuracy

Figure 6

Top 30 TFBSs selected by RF and their mean decrease in accuracy in sustained vs. transient SAMD target up-regulated genes. The mean decrease in accuracy was an average of 100 runs of RF. Experimentally known SMAD interacting TFBSs are marked with . Statistically significant ( $p$-value $<0.05$ by Fisher's exact test) over-represented TFBSs are labeled in magenta - over-represented in the sustained group as compared with the transient group.

regulation of KITLG expression has been reported in ovarian cancer [38,39]. Taken together, these observations suggest that disruption of TGF- $\beta$ signaling pathway may lead to altered KITLG expression, which in turn could contribute to ovarian cancer carcinogenesis. An exciting extension is the possibility of KITLG activation in ovarian cancer initiating cells (OCICs), as we recently reported upregulation of the KITLG receptor, c-kit/CD117, in this highly tumorigenic subpopulation of cells in human ovarian adenocarincomas [40].

It is interesting to note that TGFBR2 and SMAD3 were among the down-regulated targets, suggesting a possible 

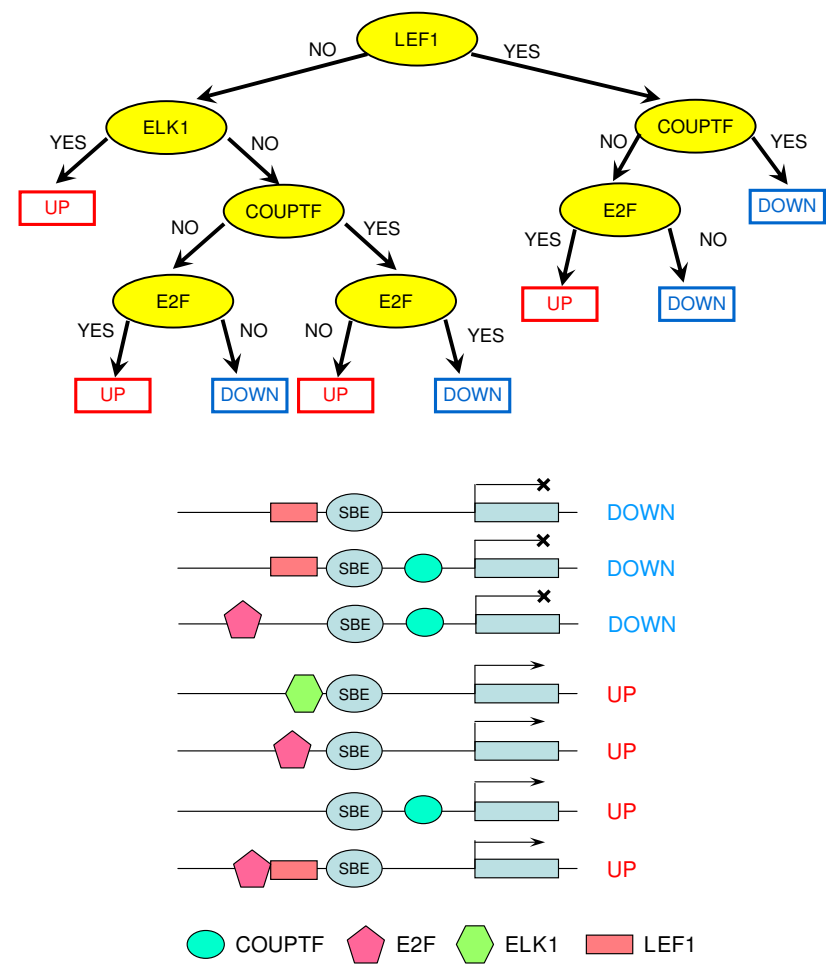

Figure 7

Classification model that discriminates up- and down-regulated TGF- $\beta$ /SMAD targets and corresponding SMAD co-regulatory modules. Upper panel shows the CART model that discriminates between the TGF- $\beta / S M A D$ up- and down-regulated targets. Lower panel shows the derived cis-regulatory modules of these target genes identified by the CART model. In the CART trees, "yes" means the TFBS was present and "no" means the TFBS was absent.

negative feedback loop in the pathway. Since our microarray platform spans only the promoter regions, the number of binding sites in the present study is likely to be an underestimate at the whole genome level as recent finding indicates that the transcription factor binding happens throughout the genome [41]. On the other hand, we have identified a total of 1946 SMAD4 targets by ChIP-Chip, but only 150 shows expression changes. The other 1796 targets, however, may show expression changes after 12 hours of TGF- $\beta$ stimulation but could not be detected in the current setting. Alternatively, those targets may require other transcription factor(s) for initiation. Although the presence of false positive cannot be excluded, our experimental design, in which the ChIP-chip data is subtracted from $3 \mathrm{hr}$ after addition of TGF- $\beta$ to $0 \mathrm{hr}$, should have minimized this possibility.

In order to decipher complex gene regulatory networks associated with signaling pathways that play critical roles
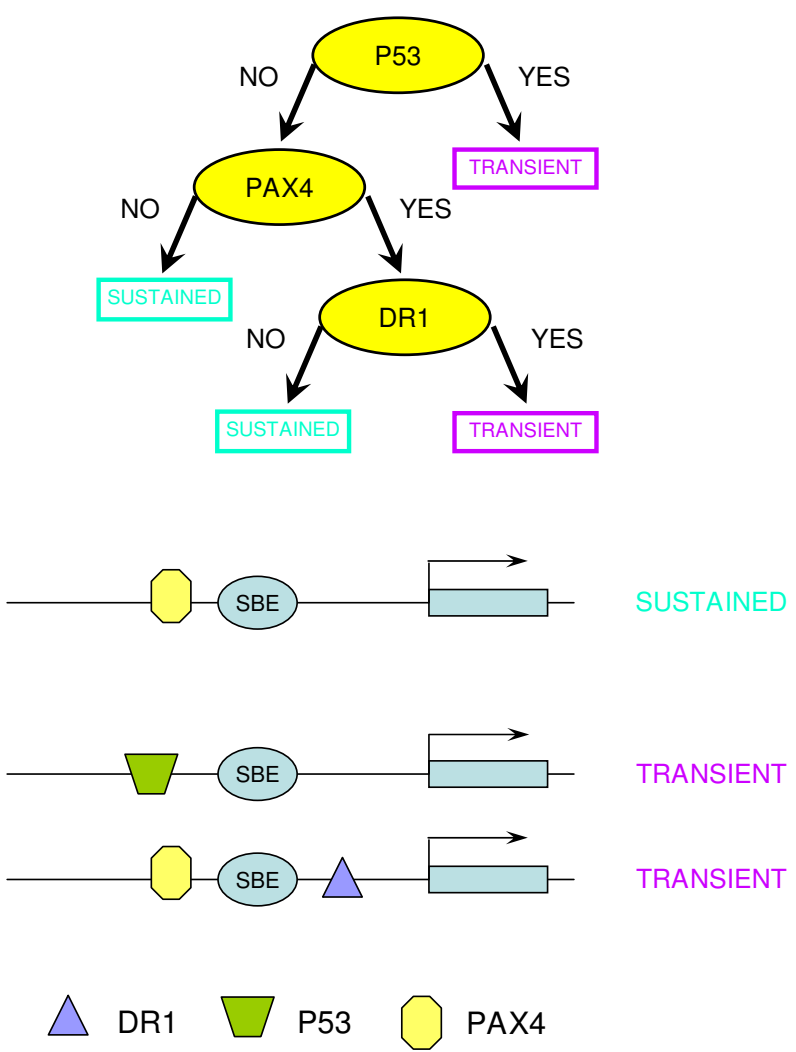

Figure 8

Classification model that discriminates sustained and transient TGF- $\beta$ /SMAD targets within the up-regulated group. Upper panel shows the CART model and lower panel shows the derived cis-regulatory modules of these target genes identified by the CART model.

in normal and aberrant cell behavior, accurate prediction of transcription factor co-regulatory modules is essential [42]. Computational analyses that rely solely on motifs derived from position weight matrix scanning are considered far from perfect and known to produce both falsepositive and false-negative results [26]. Phylogenetic footprinting, can be used to identify conserved sequences between distantly related species thereby improving module discovery [43]. However, this comparative genomics approach can only partially improve prediction accuracy due to the lack of conserved binding sites among species and the unavailability of human gene counterparts in other organisms for comparative genomic analysis. With the advance of ChIP-chip technologies, we can now computationally interrogate the interactions between cis-acting elements and transcription factors using experimental data [44]. Recently, computational approaches that combine seemingly disparate experimental data have been successful in developing concise pathway models and transcriptional modules [45,46]. RFs have been receiving 
Table 3: Functional comparison of SMAD responsive (from gene expression) and SMAD-target (from ChIP-chip) gene sets obtained using Ingenuity Core analyses

\begin{tabular}{|c|c|c|c|c|c|}
\hline \multirow[t]{2}{*}{ IPA Signaling Pathway } & \multicolumn{3}{|c|}{ Affymetrix (1095 genes) } & \multicolumn{2}{|c|}{ SMAD predicted (I50 targets) } \\
\hline & IPA ratio & IPA p-value & No. molecules & IPA ratio & IPA molecules \\
\hline TGF-beta signaling & 0.169 & $3.68 \mathrm{E}-06$ & 14 & 0.012 & SMAD3 \\
\hline RAR activation & 0.08 & $2.50 \mathrm{E}-03$ & 15 & 0.011 & GTF2H2, SMAD3 \\
\hline IGF-I signaling & 0.108 & $3.58 \mathrm{E}-03$ & 10 & $\mathrm{n} / \mathrm{a}$ & $\mathrm{n} / \mathrm{a}$ \\
\hline Wnt/beta-catenin signaling & 0.084 & 4. I2E-03 & 14 & 0.018 & CSNKIAI, DKKI, FRATI \\
\hline Cell cycle: GI/S checkpoint regulation & 0.117 & 7.37E-03 & 7 & 0.033 & HDAC9, SMAD3 \\
\hline BMP signaling & 0.097 & I.0IE-02 & 10 & $\mathrm{n} / \mathrm{a}$ & $\mathrm{n} / \mathrm{a}$ \\
\hline GM-CSF signaling & 0.113 & I.34E-02 & 7 & 0.016 & CAMK2D \\
\hline LPS/IL-I mediated inhibition of RXR function & 0.065 & $2.68 \mathrm{E}-02$ & 13 & $\mathrm{n} / \mathrm{a}$ & $\mathrm{n} / \mathrm{a}$ \\
\hline IL-4 signaling & 0.1 & $5.15 \mathrm{E}-02$ & 7 & 0.014 & NFAT5 \\
\hline
\end{tabular}

Only significant signaling pathways are listed. Ratio shows the relative overlap between number of molecules per pathway within each dataset over the total number of pathway-specific molecules present in Ingenuity Knowledge Base.

increased attention in the data-mining field as a means of variable selection in many classification tasks in computational biology, including the selection of a subset of genetic markers and genes in microarray data analysis relevant for the prediction of a certain disease [47-50]. Here, we have used an integrative modeling approach that combines CART and RF to classify different SMAD target promoters with reasonably good classification accuracy and reduced instability. Other popular classification methods, such as Naïve Bayes Tree, Logistic Model Tree, Bagging and LogitBoost (reviewed in [51]) or combination of these algorithms with RF may give different performance results and derive different SMAD modules, which needs a systematic testing. Although the main goal in classification is to build a model with minimal mis-classification error in cross-validation (Table 1), in this application we are equally interested in identifying TFBSs as highly important discriminating variables. One of the main goals of our analyses is to select potential SMAD interacting transcription factors from a large feature space $(>150$ transcription factors from Transfac database) in order to build SMAD modules. RF algorithm generates internal estimates of the decrease in the classifier's overall accuracy if that particular variable was not used in building the classifier. Thus, variables (TF binding sites) with larger importance measures can be deemed to have more power in discriminating different groups. A notable fact about our $\mathrm{RF}$ feature selection procedure is that more than one third of the transcription factors in the top ranking variables (Figures 5 and 6) are previously known to synergistically interact with SMAD in regulating the target promoter (Table 2). Conversely, a substantial number of the known SMAD co-regulators appeared as the most important variables (See Additional File 5 - Table S4). This demonstrates the power of RF feature selection procedure and indicates that other top ranking transcription factors could be novel partners of SMAD, resulting in different transcriptional outcomes.

We first built a large number of RFs to identify and rank TFBSs of importance; and then supplied the resultant TFBSs as a relatively smaller set of predictor variables to CART for classification, using step-wise forward selection procedure. Based on our original microarray data, this process dramatically improved the misclassification error rate compared to using CART or RF analysis alone. By running a large number of RFs, we obtained a stable rank for the most important variables, which could not be achieved with a single RF run. When fitting the CART model, a series of models were built, starting with the most important TFBS as the predictor variable, followed by systematically adding more TFBSs from the variable reservoir. As expected, the overall misclassification error rate (defined as the sum of the error rates for the individual groups) first decreased and then increased again (See Additional File 8 - Figure S5). The one at the bottom of 


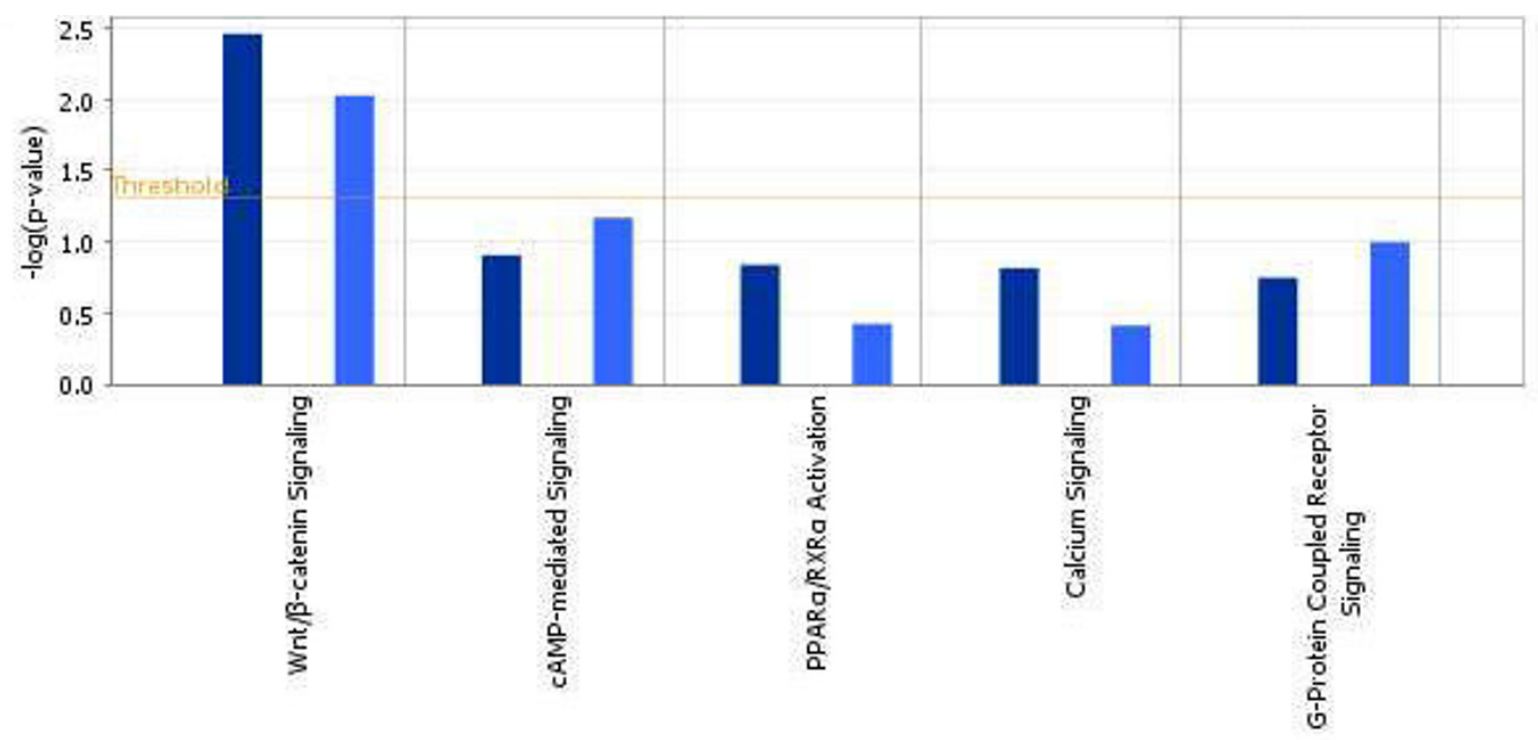

() 2000-2008 Ingenuity Systems, Inc. All rights reserved.

B

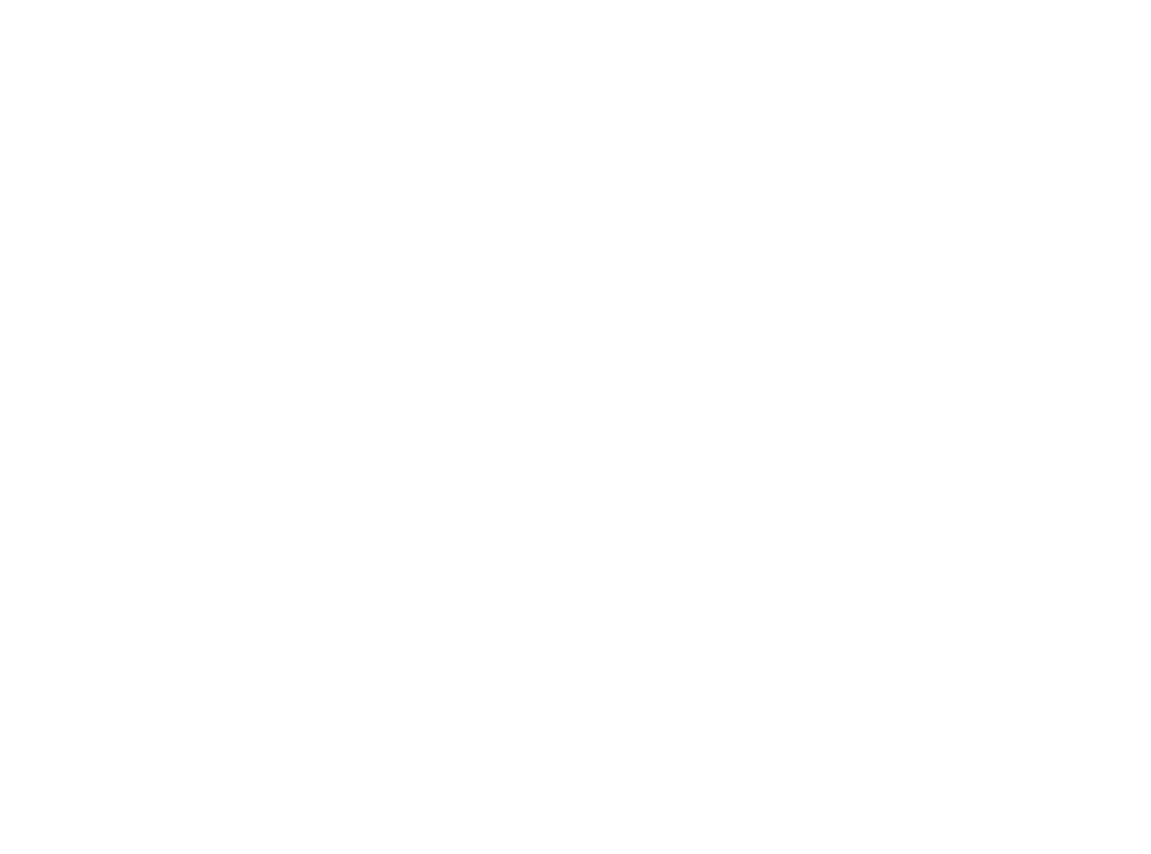

(c) 2000-2008 Ingenuity Systems, Inc. All rights reserved.

\section{Figure 9}

Ingenuity Pathway Analysis of SMAD targets: (A) A graphical representation of overlapping signaling pathways detected in differentially expressed genes and SMAD-target genes. Wnt/ $\beta$-catenin was found to be significant. (B) The result of Ingenuity analyses of Affymerix gene expression and SMAD-predicted datasets. There are total nine significant signaling pathways (shown in bar graphs) for gene expression data, and six significant signaling pathways for SMAD-predicted molecules (shown by asterisks). There is a major functional overlap (about $70 \%$ or $6 / 9$ ) between gene expression and SMAD-predicted data. Note that the $p$ values were calculated by Ingenuity algorithm http://www.ingenuity.com per each pathway within Affymerix gene expression dataset and minus $\log P$ values are shown. The minus $\log P$ values for SMAD-predicted molecules are not shown. 
the decreasing trend is the best model, overcoming the limitation of using pre-set arbitrary cutoff values for variable selection in other CART models [42].

By computational prediction, $83 \%$ of the target promoters contained SMAD4 consensus sequences, a significant enrichment compared to a random set of sequences. The consensus sequence of the SBE, nevertheless, contains only a weak signal. To ensure the binding of the SMAD complex, the presence of the binding sites of the co-regulators is equally important. Based on the ChIP-chip data, the combined classification tree analysis accurately predicted previously known TGF- $\beta / S M A D$ co-regulators, including LEF1 [52], ELK1 [53], COUPTF [54], E2F [55], and P53 [56]. The transcription factors that recognize the DR1 site, PPAR, HNF-4, COUPTF and RAR are all known SMAD partners [54,57-59]. The combined RF and CART analysis also uncovered a novel co-regulator, PAX4, a paired-homeodomain transcription factor and important regulator of pancreas development [60,61]. Previous studies have demonstrated regulation of PAX4 expression by activin A, a TGF- $\beta$ superfamily member, and transcriptional regulation via interactions between paired domain transcription factors PAX8 and PAX6 and SMAD $[62,63]$. Therefore, it seems reasonable to suggest that our computational prediction of a PAX4-SMAD interaction and subsequent target gene co-binding could contribute to gene up-regulation (Table S5). Furthermore, as a potential tumor suppressive function for PAX4 has recently been reported [64], we speculate that disruption of PAX4 could compromise TGF- $\beta$-mediated growth inhibition and contribute to ovarian carcinogenesis.

Our integrative computational modeling and Ingenuity Pathway Analysis suggests that SMAD and LEF1 co-regulate some of the up-regulated SMAD responsive genes. It was shown that the activation of MSX2 gene was mediated via the cooperative binding of SMAD4 at two SBEs and of LEF1 at two Lef1/TCF binding sites [65]. However, these predicted SMAD regulatory modules need to be confirmed by biological experiments. First, quantitative ChIPPCR with the antibody against a TF can corroborate the recruitment of the TF to the promoter region of the target gene. Second, a promoter of a target gene with a TFBS deleted can be compared to a wild-type promoter to see if the TFBS confers any biological activity in a promoterreporter assay setting. Third, comparison of target gene expression levels in cells transfected with siRNAs against specific SMAD proteins, or against a TF predicted to be a SMAD co-regulator, or against both, can reveal if there is any synergistic action between the two interacting partners.

\section{Conclusion}

In conclusion, we have identified 124 TGF- $\beta /$ SMAD targets by microarray experiments coupled with bioinfor- matics. Further computational analysis using CART and RF modeling has identified several transcription factor modules and provided important information in the transcriptional control of TGF- $\beta / S M A D$ signaling. Guided by this computational information, experiments are underway to verify these co-regulator modules in normal and aberrant conditions such as ovarian cancer, a disease in which dysregulation of TGF- $\beta / S M A D$ signaling has recently been demonstrated [66].

\section{Methods}

\section{Chromatin Immunoprecipitation Microarray (ChIP-chip)}

IOSE cells were maintained in a 1:1 mixture of medium 199 (Sigma, St. Louis, MO) and 105 (Sigma) supplemented with $10 \%$ FBS, $400 \mathrm{ng} / \mathrm{ml}$ hydrocortisone (Sigma), $10 \mathrm{ng} / \mathrm{ml}$ EGF and 50 units/ml of penicillin/ streptomycin (Invitrogen, Carlsbad, CA). The cells were treated with $10 \mathrm{ng} / \mathrm{ml}$ of TGF- $\beta 1$ (Sigma) for $3 \mathrm{hrs}$ and then crosslinked with $1 \%$ formaldehyde for $10 \mathrm{~min}$. Chromatin immunoprecipitation was performed by using a ChIP assay kit (Upstate Biotechnology, Lake Placid, NY) as described previously [42]. In brief, $1 \times 10^{6}$ cells were sonicated and immunoprecipitated by incubation with an anti-SMAD4 polyclonal antibody (H552, Santa Cruz Biotechnology, Santa Cruz, CA). Immunoprecipitated (ChIP) and total input DNAs were amplified by ligation-mediated-PCR for 24 cycles. Two $\mu \mathrm{g}$ of ChIP-DNA or input DNA were aminoallyl-labeled using the BioPrime DNA labeling system (Invitrogen) and then coupled with Cy5 and $\mathrm{Cy} 3$ fluorescence dyes (Amersham, Buckinghamshire, UK), respectively. DNAs were then co-hybridized to a modified version of whole genome oligonucleotide microarray ( 44,000 60-mer probes; Agilent Technologies, Palo Alto, CA) representing the promoter regions of $\sim 17,000$ unique genes [67]. Following hybridization, the arrays were washed and then scanned using a GenePix 4000A Scanner (Axon, Union City, CA), followed by analysis with GenePix Pro 6.0 software (Axon).

\section{Expression Microarray}

Total RNAs from IOSE cells treated with TGF- $\beta 1$ at $0,3,6$, and 12 hrs were extracted using TRIzol reagent (Invitrogen) following the manufacturer's instructions. In brief, about $1.5 \mu \mathrm{g}$ of total RNA were reverse transcribed into cDNA using a HPLC-purified T7-oligo(dT) primer and Superscript II reverse transcriptase (Invitrogen). Biotinylated antisense cRNAs were then generated by in vitro transcription using the Bioarray RNA transcript labeling kit (Enzo Life Sciences, Farmingdale, NY). Fifteen $\mu \mathrm{g}$ of fragmented cRNAs were then hybridized to a Human Genome U133A plus 2.0 GeneChip Oligonucleotide Array (Affymetrix, Santa Clara, CA), representing over 47,000 transcripts in the human genome. Finally, the microarray slides were scanned using a GeneChip Scanner 3000 (Affymetrix) and analyzed by GeneChip Operating Software. 


\section{Microarray Analysis}

For ChIP-chip, low quality spots that were flagged by the software and spots that had a percentage of pixels with intensities more than two standard deviations above the background for each color $\leq 50 \%$ and spots with signal to noise ratio of $\leq 1.25$ were excluded from the analysis. Background corrected median intensities of two channels were normalized by using intensity-dependent lowess normalization, and the binding ratio of immunoprecipitated DNA to control DNA was determined for each spot on the microarray [68]. By adopting a single-array error model, a confidence estimate ( $p$-value) for each binding ratio was estimated using standard deviations of the two background-intensities [69]. Binding ratios for each of the 2 replicated samples were combined by a weighted average method, and final $p$-values were calculated for the weighted ratios at the 0 - and 3-hr time points [69]. A gene was deemed to be bound if its weighted ratio at 3-hr time point is above 2 with $p$-value $<0.01$ and showed at least a $30 \%$ increase in relative binding compared to $0 \mathrm{hr}$ time point. Then, in order to identify candidate TGF- $\beta /$ SMAD targets from the bound gene list, genes that showed significant changes in gene expression would be selected after expression microarray analysis.

For expression microarrays, gene expression levels from probe intensities were estimated using a robust multichip average method with quantile normalization and background correction [70]. Principal component analysis (PCA) of 8 Affymetrix arrays showed high variation between the two sets of replicated arrays that were processed on two distinct days (day1 and day2). A two-way mixed model ANOVA analysis with a fixed effect of time variation ( $0 \mathrm{hr}, 3 \mathrm{hr}, 6 \mathrm{hr}$, and $12 \mathrm{hr}$ ) and a random effect of day variation (day1 and day2), using Partek software (Partek Discovery Suite 6.2, Partek Inc, St. Louis, MO), was performed to identify and remove the day-to day variation from gene expression estimates. Corrected gene expression estimates, were used for further analysis. Cluster analysis of 8 samples using all the genes, was performed using centered correlation metric and average linkage method. To study the effect of different time points on gene expression, ANOVA analysis was applied. Individual contrasts within the ANOVA model was estimated in order to identify differentially expressed genes that show significant up- or down-regulation at 3, 6 or 12 hr compared to $0 \mathrm{hr}$ using Partek software. Genes with a significant contrast $(p$-value $<0.05)$ and an absolute fold change over 1.5 were considered as differentially regulated.

Gene promoters that were identified as Smad4-bound at 3 hr (relative to $0 \mathrm{hr}$ ) in the ChIP-chip data analysis, and those that showed significantly differential gene expression estimates at $3 \mathrm{hr}, 6 \mathrm{hr}$ or $12 \mathrm{hr}$ (relative to $0 \mathrm{hr}$ ), were considered to be candidate TGF- $\beta /$ SMAD targets. Hierarchical cluster analysis of the candidate TGF- $\beta$ /SMAD target genes was then performed by the centered correlation metric and average linkage method, using expression array data [71]

\section{Quantitative ChIP-PCR and RT-PCR}

To confirm candidate TGF- $\beta / S M A D$ target promoters, ChIP-PCR was conducted using immunoprecipitated DNAs as templates. Primers (sequences available upon request) were designed to flank a region within 500 bp of the predicted SMAD binding element. Fold-enrichment of amplified DNAs was determined using previously described protocols [42].

Quantitative RT-PCR was performed as described previously [42]. To remove potential DNA contamination, $2 \mu \mathrm{g}$ of total RNA was treated with DNase I (Invitrogen) and then reverse transcribed with Superscript II reverse transcriptase (Invitrogen). Specific primers for amplification are available on request.

\section{Ingenuity Pathways Analysis}

Ingenuity Pathways Analysis (Ingenuity ${ }^{\circledR}$ Systems, http:// www.ingenuity.com, IPA 6.0) Compare Biomarker feature was used to define common molecules for gene expression (Affymetrix U133A, 1095 molecules) and ChIP-chip data (Agilent Technologies, 2135 molecules) followed by functional analyses aimed to determine the similarities between SMAD-module predicted and IPAgenerated targets. The functional analyses run separately on SMAD-predicted and IPA-generated targets identified the biological functions that were most significant to the data sets. Genes from each data set associated with biological functions in the Ingenuity Pathways Knowledge Base (IPKB) were considered for analyses. In addition, IPA Core analysis feature was used to define the signaling pathways common for Affy and SMAD-predicted datasets. More than 800,000 objects currently present in IPKB ontology were used to define algorithmically whether the tested dataset fit into the existing canonical pathways. Fischer's exact test was used to calculate a p-value determining the probability that each biological function assigned to that data set is due to chance alone. Ratios were the relative representation of the number of hits within the tested dataset against the total number of hits within the IPKB.

\section{Prediction of SMAD and Other Transcription Factor Binding Sites}

Sixty-seven SMAD binding elements (SBEs) from human, mouse and rat were collected from the published literature (See Additional File 9 - Table S6). The consensus SBE 5'-CAGAC-3' was extended 6 bp upstream and 4 bp downstream. A SBE position weight matrix on the $15 \mathrm{bp}$ 
sequences was constructed by formula
$w(b, i)=\log _{2} \frac{p(b, i)}{p(b)}=\log _{2} \frac{\left(f_{b, i}+\sqrt{N} / 4\right) /(N+\sqrt{N})}{p(b)}$, where $f_{b}$ ${ }_{i}$ is the number of nucleotide $b$ at position $i, N$ is the number of sequences, and $p(b)$ is the background frequency of nucleotide $b$. In the PWM, the $8^{\text {th }}$ and $9^{\text {th }}$ positions were fixed to nucleotide $A$ and $G$, respectively. The background frequency was determined on 100,000 pseudo-SBE sequences. A 3,000 bp region $(-2,000 \mathrm{bp}$, $+1,000 \mathrm{bp})$, flanking the transcription start site of randomly selected human promoters from the MPromDb database, was extracted [72]. For every AG nucleotide base pair present in the promoter sequence, the flanking regions $(-7 \mathrm{bp},+6 \mathrm{bp})$ were extracted to represent a $15 \mathrm{bp}$ pseudo-SBE sequence. An in-house Perl script was developed that could detect SBEs in Fasta sequences (program available upon request). The core score was the score on the 5 bp consensus SBE; the full score was that on the whole 15 bp sequence. In determining SBEs, the 60-mer sequence on the $44 \mathrm{~K}$ promoter microarray was equally extended on both directions to a 1,060 bp sequence and scanned for SBE using 0.9 and 0.7 as cutoffs for the core and full scores, respectively. Other transcription factor binding sites that fall within -220 bp to +220 bp of a SBE were of interest in the CART and RF analysis. These binding sites were detected by the MATCH program, using the position weight matrices from TRANSFAC database (TRANSFAC 9.1, minSum profile) [26].

\section{Random Forest}

The Random Forest (RF) classifier is based on growing a large ensemble of classification trees, and the majority vote of the trees determines the class of an observation [21]. Each tree is constructed on a bootstrapped sample from the original data and only a number of randomly selected predictor variables are used in tree branch splitting. This number is a user-defined parameter or set to a default value, i.e. square root of the total number of predictor variables. The result is not sensitive to this number. An estimate of the classification error is supplied by the out-of-bag sample, i.e. the observations that are not used in a particular tree construction. The class label of the outof-bag sample is predicted by the tree and compared with the true label of the sample. The whole tree ensemble thus generates the misclassification error rate.

While RF is considered as a "black box" method, with no interpretable classification model is present at the end of the application, it still provides useful information, such as variable importance. One of the measures of variable importance is the mean decrease in accuracy, calculated using the out-of-bag sample. The difference between the prediction accuracy on the untouched out-of-bag sample and that on the out-of-bag sample permuted on one predictor variable is averaged over all trees in the forest and normalized by the standard error. This gives the mean decrease in accuracy of that particular predictor variable which has been permuted. Thus, the importance of the predictor variables can be ranked by their mean decrease in accuracy.

For our random forest analysis, we used the RF package in R http://www.r-project.org, with each RF run having 1000 trees in the forest. Stratified sampling with equal sample size in each stratum was employed for the bootstrapped sampling [73]. The dependent variable was the synexpression group labeling derived from the hierarchical clustering of the expression levels of the TGF- $\beta /$ SMAD target genes. Each predicted transcription factor binding site within the 440 bp sequence, which was centered on a predicted SBE, was considered as a binary predictor variable, with 1 for presence, 0 for absence. Transcription factor binding sites that were present in at least 35\% of the sequences of either class were retained in the data matrix for RF, as well as for CART analysis.

\section{Classification and Regression Tree Analysis}

Because of its simplicity and interpretability, CART is one of the most frequently used classification tools [22]. The building of the tree is a 3-stage process. In the first stage, the tree is grown by recursively dividing the data space to binary spaces. The splitting variable and the splitting point can be selected by several criteria. We used the Gini index, defined as $1-\sum_{j} p^{2}(j \mid t)$, for a node $t$ with estimated class probabilities $p(j \mid t), j=1, \ldots, J$, where $J$ is the total number of classes. Once a large tree is grown, the next step is to prune it, until the root node remains in the tree. This pruning procedure, guided by a minimal cost complexity measure, creates a nested subset of trees. The optimum tree is the one with the lowest misclassification error rate by cross validation. CART software (Salford Systems, San Diego, CA) was used in our analysis with equal prior setting and a 10 -fold cross validation. The misclassification cost for each class was set to 1 . We note that CART differs with RF in that it only builds one tree based on all predictor variables; while RF combines multiple (1000 in our case) trees that are constructed on randomly selected predictor variables.

\section{Random Forest Variable (TFBSs) Selection for CART Model}

When the number of predictors is large and the number of observations is small (so called "small $n$ large $p$ " problem in the field of Statistics), CART generally produces a poor 
classification result due the instability of the individual trees. However, RFs can increase the prediction accuracy as compared to single CART tree, because the ensemble adjusts for the instability of the individual trees induced by small changes in the learning sample, which weakens the prediction accuracy in test samples [22]. Therefore, we first used RF for selecting the most important variables, which were then included in the CART analysis. Because of the randomness inherent to $\mathrm{RF}$, such as random bootstrapping of the data and random selection of the predictor variables for splitting, the most important variables selected by RF would be different from each run. However, the variables that are truly important would consistently appear at the top of the most important list. Therefore, we ran the RF 100 times, with each run having 1000 trees. The variables were then ranked by the average of the 100 runs, with regard to the mean decrease in accuracy.

A series of CART models were then built on the top 30 most important variables in a systematic way. The dataset that the CART model was built upon, initially had only one independent variable (the most important one), and was expanded, by adding more independent variables from the list of the top 30 most important variables, in the order of decreasing importance. The error rates from cross-validation of the CART models are shown in Figure S5A (See Additional File 8). The CART model with the lowest overall misclassification error rate was chosen as the final model. This criterion also agrees with using sensitivity and specificity to judge the model performance (See Additional File 8 - Figure S5B).

The ChIP-chip data are under the accession number [GEO:GSE6727] and the Affymetrix gene expression data are under the accession number [GEO:GSE6653] at the GEO database.

\section{Abbreviations}

TGF- $\beta$ : transforming growth factor beta; SBE: SMAD Binding Element; TFBS: transcription factor binding site; ChIPchip: Chromatin Immunoprecipitation (ChIP) followed by microarray analysis; CART: Classification And Regression Tree Analysis; RF: Random Forest algorithm; IOSE: immortalized ovarian surface epithelial cells

\section{Competing interests}

The authors declare that they have no competing interests.

\section{Authors' contributions}

HQ designed the computational methods and performed the statistical analyses. MWYC designed the experimental methods and performed the ChIP-chip experiments. PY coordinated the microarray experiments. SL, DP, IJS, FJA helped with the bioinformatics analyses. CB and ASLC helped with the experimental methods. EVN performed the pathway analyses. HJL, KPN, JHS and LCS participated in the design of the study. $\mathrm{RD}$ and $\mathrm{TH}$ formulated and directed the design of the study. All authors read and approved the final manuscript.

\section{Additional material}

\section{Additional file 1}

Figure S1. Reproducibility of ChIP-chip experiments. Normalized log ratios (immonuprecipated DNA over total input DNA) of the biological replicate experiments ( 0 hrs untreated or $3 \mathrm{hrs}$ TGF- $\beta 1$-treated) are plotted as smooth scatter plots. Binding ratios for 150 significant genes are indicated by red dots. The overall correlation coefficient of each plot is also shown.

Click here for file

[http://www.biomedcentral.com/content/supplementary/17520509-3-73-S1.ppt]

\section{Additional file 2}

Figure S2. Reproducibility of expression microarrays. Dye intensities $(\log 2)$ from the technical replicate experiments (0 hrs untreated, 3, 6, 12 hrs TGF- $\beta 1$-treated) are plotted as scatter plots. Expression data for 150 significant genes are indicated by red dots. The overall correlation coefficient of each plot is also shown.

Click here for file

[http://www.biomedcentral.com/content/supplementary/17520509-3-73-S2.ppt]

\section{Additional file 3}

Figure S3. Cluster analysis of expression microarray. Data from expression microarrays were used to perform cluster analysis. The replicates at each time points were technical replicates and were labeled as "Rep1 " and "Rep2". The scale bar is "1-correlation". Therefore, the shorter the distance, the stronger the correlation. The result showed that data from the treated and the untreated experiments can be grouped into two different clusters.

Click here for file

[http://www.biomedcentral.com/content/supplementary/17520509-3-73-S3.ppt]

\section{Additional file 4}

Table S1. List of 150 putative TGF- $\beta / S M A D$ target genes and their expression levels. Genes are sorted from most change to lease change according to binding response to treatment.

Click here for file

[http://www.biomedcentral.com/content/supplementary/17520509-3-73-S4.xls]

\section{Additional file 5}

Supplementary Tables S2, S3 and S4. Table S2. Distribution of TGF- $\beta /$ $S M A D$ target genes. Table S3. Misclassification rates by CART and RF modeling with three synexpression groupsTable S4. Selection of known SMAD co-regulators by RF.

Click here for file

[http://www.biomedcentral.com/content/supplementary/17520509-3-73-S5.doc] 


\section{Additional file 6}

Table S5. Predicted modules for TGF- $\beta /$ SMAD target genes. Column 1 shows the SMAD target gene, column2 gives the predicted SMAD module; columns 3 and 4 show the predicted and observed groups respectively. Click here for file

[http://www.biomedcentral.com/content/supplementary/17520509-3-73-S6.xls]

\section{Additional file 7}

Figure S4. A graphical representation of overlapping molecular and cellular functions in SMAD responsive (from Affymetrix array data) and SMAD target (from ChIP-chip) gene sets from Ingenuity Pathway Analysis. A graphical representation of overlapping molecular and cellular functions for 73 IPA and 145 SMAD-module predicted targets sorted by a p-value. The significance of each function was calculated by Fischer's exact test (see Methods).

Click here for file

[http://www.biomedcentral.com/content/supplementary/17520509-3-73-S7.ppt]

\section{Additional file 8}

Figure S5. Choosing the best CART model by step-wise forward variable selection procedure. Figure S5A: Plot of the mean error rates as a function of the number of variables in the CART model (top ranking 30 most important variables selected by RF were used by step-wise forward selection, starting with the most important variable) for dataset 1: up- vs. down-regulated targets and dataset 2: Sustained up- vs. transient up-regulated targets. The error rates were a summation of the error rates of the two classes and were estimated from 10-fold cross-validation. The error rates first dropped and then increased as a function of the number of independent variables. The best CART models, in terms of the lowest overall error rate, consisted of 4 variables for up $v$ down and 3 variables for sustained up- vs. transient up-regulated targets. Figure S5B: The sensitivity versus 1 -specificity plot of the CART models. Down regulated target class and transient up-regulated class were selected as positive group for datasets -1 and 2, respectively. The sensitivity and specificity values were derived from the confusion matrix on the test data reported by the CART software. The point closest to the upper left corner (1-specificity $=0$, sensitivity $=1$ ) on each plot was indicated with an arrow, which was the best model in terms of a balance between sensitivity and specificity. For both datasets, equal mis-classification cost rate was used. Consequently, the model with optimal sensitivity and specificity values was also the model with the lowest overall error rate.

Click here for file

[http://www.biomedcentral.com/content/supplementary/17520509-3-73-S8.ppt]

\section{Additional file 9}

Table S6. List of 67 sequences containing SBEs from the published literature. Column 1 shows the number of experimentally known binding sites (SBEs) within each target gene; columns 2, 3, 4 and 5 give the gene symbol, Unigene ID, Accession ID and Gene ID respectively; columns 6 and 7 give the relative start and end positions of the SBE (relative to transcription start site); column 8 gives the SBE; columns 9 to 12 give the chromosomal location of the SBE (The genomic coordinates are according to Human NCBI Build 35; Rat Nov. 2004 (rn4) assembly \& Mouse NCBI Build 36); columns 13 and 14 give the sequence around SBE (-50 to +50 around the $S B E)$ and its length respectively.

Click here for file

[http://www.biomedcentral.com/content/supplementary/17520509-3-73-S9.xls]

\section{Acknowledgements}

This work was supported by National Cancer Institute grant U54 CAII $300 \mathrm{I}$ and by funds from The Ohio State University Comprehensive Cancer Center-Arthur G. James Cancer Hospital and Richard J. Solove Research Institute. D.P. is partly supported by an NIH postdoctoral fellowship to the Human Cancer Genetics Program of The Ohio State University.

\section{References}

I. Massague J, Seoane J, Wotton D: Smad transcription factors. Genes Dev 2005, 19:2783-28I0.

2. Shi $Y$, Massague J: Mechanisms of TGF-beta signaling from cell membrane to the nucleus. Cell 2003, I I 3:685-700.

3. Feng $X H$, Derynck R: Specificity and versatility in tgf-beta signaling through Smads. Annu Rev Cell Dev Biol 2005, $21: 659-693$.

4. Dennler S, Itoh S, Vivien D, ten Dijke P, Huet S, Gauthier JM: Direct binding of Smad3 and Smad4 to critical TGF beta-inducible elements in the promoter of human plasminogen activator inhibitor-type I gene. Embo J 1998, I 7:309|-3100.

5. Seoane J, Le HV, Shen L, Anderson SA, Massague J: Integration of Smad and forkhead pathways in the control of neuroepithelial and glioblastoma cell proliferation. Cell 2004, I I7:2 | I-223.

6. Boyer Arnold N, Korc M: Smad7 abrogates transforming growth factor-betal-mediated growth inhibition in COLO357 cells through functional inactivation of the retinoblastoma protein. J Biol Chem 2005, 280:21858-21866.

7. Naso M, Uitto J, Klement JF: Transcriptional control of the mouse Col7al gene in keratinocytes: basal and transforming growth factor-beta regulated expression. I Invest Dermato 2003, $121: 1469-1478$.

8. Bailey JS, Rave-Harel N, McGillivray SM, Coss D, Mellon PL: Activin regulation of the follicle-stimulating hormone beta-subunit gene involves Smads and the TALE homeodomain proteins Pbxl and Prep I. Mol Endocrinol 2004, I 8: I I58-I I 70.

9. Oren T, Torregroza I, Evans T: An Oct-I binding site mediates activation of the gata2 promoter by BMP signaling. Nucleic Acids Res 2005, 33:4357-4367.

10. de Caestecker MP, Yahata T, Wang D, Parks WT, Huang S, Hill CS, Shioda $T$, Roberts $A B$, Lechleider RJ: The Smad4 activation domain (SAD) is a proline-rich, p300-dependent transcriptional activation domain. J Biol Chem 2000, 275:2 II5-2I 22.

II. Massague J, Wotton D: Transcriptional control by the TGFbeta/Smad signaling system. Embo / 2000, 19: 1745-1754.

12. Derynck R, Akhurst RJ, Balmain A: TGF-beta signaling in tumor suppression and cancer progression. Nat Genet 200I, 29:117-129.

13. Akhurst RJ, Derynck R: TGF-beta signaling in cancer - a doubleedged sword. Trends Cell Biol 200I, I I:S44-5I.

14. Berchuck A, Rodriguez G, Olt G, Whitaker R, Boente MP, Arrick BA Clarke-Pearson DL, Bast RC Jr: Regulation of growth of normal ovarian epithelial cells and ovarian cancer cell lines by transforming growth factor-beta. Am 」 Obstet Gynecol 1992, 166:676-684.

15. Yamada SD, Baldwin RL, Karlan BY: Ovarian carcinoma cell cultures are resistant to TGF-beta I-mediated growth inhibition despite expression of functional receptors. Gynecol Oncol 1999 , 75:72-77.

16. Takakura S, Okamoto A, Saito M, Yasuhara T, Shinozaki H, Isonishi S, Yoshimura T, Ohtake Y, Ochiai K, Tanaka T: Allelic imbalance in chromosome band $18 \mathrm{q} 21$ and SMAD4 mutations in ovarian cancers. Genes Chromosomes Cancer 1999, 24:264-27I.

17. Wang D, Kanuma T, Mizunuma H, Takama F, lbuki Y, Wake N, Mogi A, Shitara Y, Takenoshita S: Analysis of specific gene mutations in the transforming growth factor-beta signal transduction pathway in human ovarian cancer. Cancer Res 2000, 60:4507-45I2.

18. Datto MB, Yu Y, Wang XF: Functional analysis of the transforming growth factor beta responsive elements in the WAFI/ Cip I/p2 I promoter. J Biol Chem 1995, 270:28623-28628.

19. Song CZ, Siok TE, Gelehrter TD: Smad4/DPC4 and Smad3 mediate transforming growth factor-beta (TGF-beta) signaling through direct binding to a novel TGF-beta-responsive element in the human plasminogen activator inhibitor-I promoter. J Biol Chem 1998, 273:29287-29290. 
20. Yagi K, Furuhashi M, Aoki H, Goto D, Kuwano H, Sugamura K, Miyazono $\mathrm{K}$, Kato M: c-myc is a downstream target of the Smad pathway. J Biol Chem 2002, 277:854-86I.

21. Breiman L: Random Forests. Machine Learning 2001, 45:5-32.

22. Breiman L: Classification and regression trees Belmont, California: Wadsworth International Group; 1984

23. Boyer LA, Lee TI, Cole MF, Johnstone SE, Levine SS, Zucker JP, Guenther MG, Kumar RM, Murray HL, Jenner RG, et al.: Core transcriptional regulatory circuitry in human embryonic stem cells. Cell 2005, I 22:947-956.

24. Blanchette M, Bataille AR, Chen X, Poitras C, Laganiere J, Lefebvre C, Deblois G, Giguere V, Ferretti V, Bergeron D, et al: Genome-wide computational prediction of transcriptional regulatory modules reveals new insights into human gene expression. Genome Res 2006, 16:656-668.

25. Philippakis AA, Busser BW, Gisselbrecht SS, He FS, Estrada B, Michelson AM, Bulyk ML: Expression-guided in silico evaluation of candidate cis regulatory codes for Drosophila muscle founder cells. PLoS Comput Biol 2006, 2:e53.

26. Kel AE, Gossling E, Reuter I, Cheremushkin E, Kel-Margoulis OV, Wingender E: MATCH: A tool for searching transcription factor binding sites in DNA sequences. Nucleic Acids Res 2003, 3 I:3576-3579.

27. Foley J, Wysolmerski JJ, Broadus AE, Philbrick WM: Parathyroid hormone-related protein gene expression in human squamous carcinoma cells is repressed by mutant isoforms of p53. Cancer Res 1996, 56:4056-4062.

28. Wang J, Shou J, Chen X: Dickkopf-I, an inhibitor of the Wnt signaling pathway, is induced by p53. Oncogene 2000, 19:1843-1848.

29. Bartke T, Siegmund D, Peters N, Reichwein M, Henkler F, Scheurich $P$, Wajant $H$ : p53 upregulates cFLIP, inhibits transcription of NF-kappaB-regulated genes and induces caspase-8-independent cell death in DLD-I cells. Oncogene 200I, 20:57|-580.

30. Chan MW, Huang YW, Hartman-Frey C, Kuo CT, Deatherage D, Qin $\mathrm{H}$, Cheng AS, Yan PS, Davuluri RV, Huang TH, et al.: Aberrant transforming growth factor betal signaling and SMAD4 nuclear translocation confer epigenetic repression of ADAMI 9 in ovarian cancer. Neoplasia 2008, 10:908-919.

31. Charron F, Tessier-Lavigne M: The Hedgehog, TGF-beta/BMP and Wnt families of morphogens in axon guidance. Adv Exp Med Biol 2007, 62 1: I 16-133.

32. Hochheimer A, Tjian R: Diversified transcription initiation complexes expand promoter selectivity and tissue-specific gene expression. Genes Dev 2003, I 7:1309-1320.

33. Howard ML, Davidson EH: cis-Regulatory control circuits in development. Dev Biol 2004, 27 I:109-1 I8.

34. Baldwin RL, Tran H, Karlan BY: Loss of c-myc repression coincides with ovarian cancer resistance to transforming growth factor beta growth arrest independent of transforming growth factor beta/Smad signaling. Cancer Res 2003 , 63:1413-1419.

35. Hu G, Jain K, Hurle M: Revealing transforming growth factorbeta signaling transduction in human kidney by gene expression data mining. Omics 2005, 9:266-280.

36. Yang $M$, Nelson D, Funakoshi $Y$, Padgett RW: Genome-wide microarray analysis of TGFbeta signaling in the Drosophila brain. BMC Dev Biol 2004, 4:I4.

37. Ismail RS, Cada M, Vanderhyden BC: Transforming growth factor-beta regulates Kit ligand expression in rat ovarian surface epithelial cells. Oncogene 1999, I8:4734-474I.

38. Parrott JA, Mosher R, Kim G, Skinner MK: Autocrine interactions of keratinocyte growth factor, hepatocyte growth factor, and kit-ligand in the regulation of normal ovarian surface epithelial cells. Endocrinology 2000, I 41:2532-2539.

39. Wilczynski SP, Chen YY, Chen W, Howell SB, Shively JE, Alberts DS: Expression and mutational analysis of tyrosine kinase receptors c-kit, PDGFRalpha, and PDGFRbeta in ovarian cancers. Hum Pathol 2005, 36:242-249.

40. Zhang S, Balch C, Chan MW, Lai HC, Matei D, Schilder JM, Yan PS, Huang TH, Nephew KP: Identification and characterization of ovarian cancer-initiating cells from primary human tumors. Cancer Res 2008, 68:4311-4320.

4I. Carroll JS, Meyer CA, Song J, Li W, Geistlinger TR, Eeckhoute J, Brodsky AS, Keeton EK, Fertuck KC, Hall GF, et al.: Genome-wide analysis of estrogen receptor binding sites. Nat Genet 2006, 38: $1289-1297$.
42. Cheng AS, Jin VX, Fan M, Smith LT, Liyanarachchi S, Yan PS, Leu YW, Chan MW, Plass C, Nephew KP, et al.: Combinatorial analysis of transcription factor partners reveals recruitment of c-MYC to estrogen receptor-alpha responsive promoters. Mol Cell 2006, 21 :393-404.

43. Grad YH, Roth FP, Halfon MS, Church GM: Prediction of similarly acting cis-regulatory modules by subsequence profiling and comparative genomics in Drosophila melanogaster and D. pseudoobscura. Bioinformatics 2004, 20:2738-2750.

44. Ren B, Robert F, Wyrick JJ, Aparicio O, Jennings EG, Simon I, Zeitlinger J, Schreiber J, Hannett N, Kanin E, et al.: Genome-wide location and function of DNA binding proteins. Science 2000, 290:2306-2309.

45. Vilar JM, Jansen R, Sander C: Signal processing in the TGF-beta superfamily ligand-receptor network. PLoS Comput Biol 2006, 2:e3.

46. Lemmens K, Dhollander T, De Bie T, Monsieurs P, Engelen K, Smets B, Winderickx J, De Moor B, Marchal K: Inferring transcriptional modules from ChIP-chip, motif and microarray data. Genome Biol 2006, 7:R37.

47. Bureau A, Dupuis J, Falls K, Lunetta KL, Hayward B, Keith TP, Van Eerdewegh P: Identifying SNPs predictive of phenotype using random forests. Genet Epidemiol 2005, 28: |7| - I82.

48. Lunetta KL, Hayward LB, Segal J, Van Eerdewegh P: Screening large-scale association study data: exploiting interactions using random forests. BMC Genet 2004, 5:32.

49. Pang $H$, Lin A, Holford M, Enerson BE, Lu B, Lawton MP, Floyd E, Zhao $\mathrm{H}$ : Pathway analysis using random forests classification and regression. Bioinformatics 2006, 22:2028-2036.

50. Cutler A, Stevens JR: Random forests for microarrays. Methods Enzymol 2006, 4 I I:422-432.

51. Lerner B, D Lawrence N: A Comparison of State-of-the-Art Classification Techniques with Application to Cytogenetics. Neural Computing \& Applications 2001, 10:39-47.

52. Labbe E, Letamendia A, Attisano L: Association of Smads with lymphoid enhancer binding factor I/T cell-specific factor mediates cooperative signaling by the transforming growth factor-beta and wnt pathways. Proc Natl Acad Sci USA 2000, 97:8358-8363.

53. Miyazaki Y, Tsukazaki T, Hirota $Y$, Yonekura A, Osaki M, Shindo $H$, Yamashita S: Dexamethasone inhibition of TGF beta-induced cell growth and type II collagen mRNA expression through ERK-integrated AP-I activity in cultured rat articular chondrocytes. Osteoarthritis Cartilage 2000, 8:378-385

54. Calonge MJ, Seoane J, Massague J: Opposite Smad and chicken ovalbumin upstream promoter transcription factor inputs in the regulation of the collagen VII gene promoter by transforming growth factor-beta. I Biol Chem 2004, 279:23759-23765

55. Chen CR, Kang Y, Siegel PM, Massague J: E2F4/5 and $p I 07$ as Smad cofactors linking the TGFbeta receptor to c-myc repression. Cell 2002, I 10:19-32.

56. Wilkinson DS, Ogden SK, Stratton SA, Piechan JL, Nguyen TT, Smulian GA, Barton MC: A direct intersection between p53 and transforming growth factor beta pathways targets chromatin modification and transcription repression of the alphafetoprotein gene. Mol Cell Biol 2005, 25:1200-1212.

57. Zheng S, Chen A: Disruption of transforming growth factorbeta signaling by curcumin induces gene expression of peroxisome proliferator-activated receptor-gamma in rat hepatic stellate cells. Am J Physiol Gastrointest Liver Physiol 2007, 292:GI|3-I23.

58. Chou WC, Prokova V, Shiraishi K, Valcourt U, Moustakas A, Hadzopoulou-Cladaras M, Zannis VI, Kardassis D: Mechanism of a transcriptional cross talk between transforming growth factorbeta-regulated Smad3 and Smad4 proteins and orphan nuclear receptor hepatocyte nuclear factor-4. Mol Biol Cell 2003, I 4:1279-1294.

59. Pendaries V, Verrecchia F, Michel S, Mauviel A: Retinoic acid receptors interfere with the TGF-beta/Smad signaling pathway in a ligand-specific manner. Oncogene 2003, 22:82 I 2-8220.

60. Smith AD, Sumazin P, Das D, Zhang MQ: Mining ChIP-chip data for transcription factor and cofactor binding sites. Bioinformatics 2005, 2 I (Suppl I):i403-4I 2.

61. Smith SB, Ee HC, Conners JR, German MS: Paired-homeodomain transcription factor PAX4 acts as a transcriptional repressor in early pancreatic development. Mol Cell Biol 1999, | 9:8272-8280. 
62. Costamagna E, Garcia B, Santisteban P: The functional interaction between the paired domain transcription factor Pax8 and Smad3 is involved in transforming growth factor-beta repression of the sodium/iodide symporter gene. J Biol Chem 2004, 279:3439-3446.

63. Grocott T, Frost V, Maillard M, Johansen T, Wheeler GN, Dawes LJ, Wormstone IM, Chantry A: The MHI domain of Smad3 interacts with Pax6 and represses autoregulation of the Pax6 P I promoter. Nucleic Acids Res 2007, 35:890-90I.

64. Hata S, Hamada J, Maeda K, Murai T, Tada M, Furukawa H, Tsutsumida A, Saito A, Yamamoto Y, Moriuchi T: PAX4 has the potential to function as a tumor suppressor in human melanoma. Int Oncol 2008, 33:1065-107|.

65. Hussein SM, Duff EK, Sirard C: Smad4 and beta-catenin co-activators functionally interact with lymphoid-enhancing factor to regulate graded expression of Msx2. J Biol Chem 2003, 278:48805-488|4

66. Sunde JS, Donninger H, Wu K, Johnson ME, Pestell RG, Rose GS, Mok SC, Brady J, Bonome T, Birrer MJ: Expression Profiling Identifies Altered Expression of Genes That Contribute to the Inhibition of Transforming Growth Factor-\{beta\} Signaling in Ovarian Cancer. Cancer Res 2006, 66:8404-84I2.

67. Lee TI, Jenner RG, Boyer LA, Guenther MG, Levine SS, Kumar RM, Chevalier B, Johnstone SE, Cole MF, Isono K, et al.: Control of developmental regulators by Polycomb in human embryonic stem cells. Cell 2006, 125:30I-3I3.

68. Yang YH, Dudoit S, Luu P, Lin DM, Peng V, Ngai J, Speed TP: Normalization for cDNA microarray data: a robust composite method addressing single and multiple slide systematic variation. Nucleic Acids Res 2002, 30:e I5.

69. Hughes TR, Marton MJ, Jones AR, Roberts CJ, Stoughton R, Armour CD, Bennett HA, Coffey E, Dai H, He YD, et al.: Functional discovery via a compendium of expression profiles. Cell 2000, 102:109-126.

70. Irizarry RA, Bolstad BM, Collin F, Cope LM, Hobbs B, Speed TP: Summaries of Affymetrix GeneChip probe level data. Nucleic Acids Res 2003, 31 :el 5.

7I. Eisen MB, Spellman PT, Brown PO, Botstein D: Cluster analysis and display of genome-wide expression patterns. Proc Nat Acad Sci USA 1998, 95:| 4863-| 4868.

72. Sun H, Palaniswamy SK, Pohar TT, Jin VX, Huang TH, Davuluri RV: MPromDb: an integrated resource for annotation and visualization of mammalian gene promoters and ChIP-chip experimental data. Nucleic Acids Res 2006, 34:D98-103.

73. Gentleman R: R Programming for Bioinformatics Boca Raton, FL: CRC Press Taylor \& Francis Group; 2008.

74. Belaguli NS, Zhang M, Rigi M, Aftab M, Berger DH: Cooperation between GATA4 and TGF-beta signaling regulates intestinal epithelial gene expression. Am J Physiol Gastrointest Liver Physiol 2007, 292:GI520-1533.

75. Anttonen M, Parviainen $H$, Kyronlahti A, Bielinska M, Wilson DB Ritvos O, Heikinheimo M: GATA-4 is a granulosa cell factor employed in inhibin-alpha activation by the TGF-beta pathway. J Mol Endocrinol 2006, 36:557-568.

76. Gomis RR, Alarcon C, Nadal C, Van Poznak C, Massague J: C/EBP. beta at the core of the TGFbeta cytostatic response and its evasion in metastatic breast cancer cells. Cancer Cell 2006, 10:203-214

77. Gomis RR, Alarcon C, He W, Wang Q, Seoane J, Lash A, Massague J: A FoxO-Smad synexpression group in human keratinocytes. Proc Natl Acad Sci USA 2006, 103:12747-12752.

78. Williams JG: STAT signalling in cell proliferation and in development. Curr Opin Genet Dev 2000, I0:503-507.

79. Long J, Wang G, Matsuura I, He D, Liu F: Activation of Smad transcriptional activity by protein inhibitor of activated STAT3 (PIAS3). Proc Natl Acad Sci USA 2004, I 0 I:99- 104.

80. Nakashima K, Yanagisawa M, Arakawa H, Kimura N, Hisatsune T, Kawabata M, Miyazono K, Taga T: Synergistic signaling in fetal brain by STAT3-SmadI complex bridged by $\mathrm{p} 300$. Science 1999, 284:479-482.

81. Lim SK, Hoffmann FM: Smad4 cooperates with lymphoid enhancer-binding factor $I / T$ cell-specific factor to increase $c$ myc expression in the absence of TGF-beta signaling. Proc Natl Acad Sci USA 2006, I 03: 18580-18585.

82. Chakladar A, Dubeykovskiy A, Wojtukiewicz LJ, Pratap J, Lei S, Wan TC: Synergistic activation of the murine gastrin promoter by oncogenic Ras and beta-catenin involves SMAD recruitment. Biochem Biophys Res Commun 2005, 336: 190-196.

83. Labbe E, Lock L, Letamendia A, Gorska AE, Gryfe R, Gallinger S, Moses HL, Attisano L: Transcriptional cooperation between the transforming growth factor-beta and Wnt pathways in mammary and intestinal tumorigenesis. Cancer Res 2007, 67:75-84.

84. Callis TE, Cao D, Wang DZ: Bone morphogenetic protein signaling modulates myocardin transactivation of cardiac genes. Circ Res 2005, 97:992-1000.

85. Cordenonsi M, Montagner M, Adorno M, Zacchigna L, Martello G, Mamidi A, Soligo S, Dupont S, Piccolo S: Integration of TGF-beta and Ras/MAPK signaling through p53 phosphorylation. Science 2007, 315:840-843.

86. Furumatsu T, Tsuda M, Taniguchi N, Tajima Y, Asahara H: Smad3 induces chondrogenesis through the activation of SOX9 via CREB-binding protein/p300 recruitment. J Biol Chem 2005, 280:8343-8350.

87. Irvine SA, Foka P, Rogers SA, Mead JR, Ramji DP: A critical role for the Spl-binding sites in the transforming growth factorbeta-mediated inhibition of lipoprotein lipase gene expression in macrophages. Nucleic Acids Res 2005, 33: I 423-I434.

88. Jungert $K$, Buck $A$, von Wichert $G$, Adler G, Konig A, Buchholz M, Gress TM, Ellenrieder $V$ : SpI is required for transforming growth factor-beta-induced mesenchymal transition and migration in pancreatic cancer cells. Cancer Res 2007, 67:1563-1570.

89. Van Beek JP, Kennedy L, Rockel JS, Bernier SM, Leask A: The induction of CCN2 by TGFbetal involves Ets-I. Arthritis Res Ther 2006, 8:R36.

90. Park JI, Lee MG, Cho K, Park BJ, Chae KS, Byun DS, Ryu BK, Park YK Chi SG: Transforming growth factor-betal activates interleukin-6 expression in prostate cancer cells through the synergistic collaboration of the Smad2, p38-NF-kappaB, JNK, and Ras signaling pathways. Oncogene 2003, 22:43I4-4332.

91. Sanchez-Elsner T, Botella LM, Velasco B, Corbi A, Attisano L, Bernabeu $C$ : Synergistic cooperation between hypoxia and transforming growth factor-beta pathways on human vascular endothelial growth factor gene expression. J Biol Chem 200I, 276:38527-38535.

92. Rodriguez-Pascual F, Redondo-Horcajo M, Lamas S: Functional cooperation between Smad proteins and activator protein-I regulates transforming growth factor-beta-mediated induction of endothelin-I expression. Circ Res 2003, 92: I288- 1295.

93. Grinberg AV, Kerppola T: Both Max and TFE3 cooperate with Smad proteins to bind the plasminogen activator inhibitor-I promoter, but they have opposite effects on transcriptional activity. J Biol Chem 2003, 278: I I 227- I I 236.

94. Funaba M, Ikeda T, Murakami M, Ogawa K, Tsuchida K, Sugino H, Abe $M$ : Transcriptional activation of mouse mast cell Protease-7 by activin and transforming growth factor-beta is inhibited by microphthalmia-associated transcription factor. J Biol Chem 2003, 278:52032-5204I.

95. Cao Y, Knochel S, Donow C, Miethe J, Kaufmann E, Knochel W: The POU factor Oct-25 regulates the Xvent-2B gene and counteracts terminal differentiation in Xenopus embryos. I Bio Chem 2004, 279:43735-43743.

Publish with Bio Med Central and every scientist can read your work free of charge

"BioMed Central will be the most significant development for disseminating the results of biomedical research in our lifetime. "

Sir Paul Nurse, Cancer Research UK

Your research papers will be:

- available free of charge to the entire biomedical community

- peer reviewed and published immediately upon acceptance

- cited in PubMed and archived on PubMed Central

- yours - you keep the copyright
BioMedcentral 